\title{
QUALITÉ DU BOIS ET LARGEUR D'ACCROISSEMENTS EN FORETT DE TRONÇAIS
}

\author{
H. POLGE et R. KELLER \\ Station de Recherches sur la Qualité des Bois, \\ Centre national de Recherches forestières, I,N.R.A., \\ Champenoux 54370 Einville
}

\begin{abstract}
RÉSUMÉ
Une ètude portant sur 125 échantillons non destructifs récoltés dans 5 parcelles différentes et sur 740 éprouvettes normalisées a permis de mettre en évidence les résultats suivants :

Quand on compare entre elles les valeurs moyennes des différentes parcelles, la densité du bois augmente avec la largeur des accroissements (du fait principalement d'un double gradient négatif lié à I'àge).

A l'intérieur d'une méme parcelle, il n'y a pas de liaison significative entre densité et largeur de cernes: il est done toujours possible de trouver des arbres à croissance rapide, mais à bois tendre. Chaque fois que des accroissements larges succèdent à des aceroissements fins, la densité du bois augmente.

Les diverses mesures faites sur échantillons non destructifs et sur éprouvettes normalisées ont montré que la densité du bois est liée positivement, aussi bien aux divers critéres de résistance mécanique qu'au retrait.

La production de chẻnes de qualitè pour l'industric du tranchage ou pour l'ébénisteric fine nécessitant du bois à faible dureté et à faible rétractibilitê, on doit donc, dans des forêts comme celle de Tronçais, s'en tenir à une sylviculture relativement prudente et éviter tout à-coup dans la croissance; en outre, des programmes d'amélioration peuvent être mis sur pied pour tenter de produire dans le futur des chènes à croissance relativement rapide et à faible dureté du bois.
\end{abstract}

\section{INTRODUCTION}

Le problème des relations entre largeur des accroissements et qualité du bois de chêne préoccupe depuis longtemps et à juste titre les forestiers.

Produire plus vite des chênes plus gros pourrait être un objectif d'une grande portée économique si une accélération de la croissance était sans effet, ou même n'avait qu'une faible incidence sur les caractéristiques technologiques.

DUCELlter déjà (1930 et 1931) avait conçu un système d'éclaircies relativement fortes 
qui, accompagnées d'un repérage précoce des arbres d'avenir, devaient, dans son esprit, fournir en fin de révolution une centaine de très gros chênes à l'hectare.

Plus récemment, LORNE (1956 et 1959) a préconisé à partir de 75 ans la réalisation d' " éclaircies positives ", véritables coupes de " sélection et de grossissement ", et a établi à cet effet des tables de production orientées vers l'obtention d'accroissements annuels de $2 \mathrm{~mm}$ permettant d'obtenir vers 220 ans des chênes de $80 \mathrm{~cm}$ de diamètre.

Beaucoup se souviennent encore des discussions passionnées qui l'opposèrent par R.F.F. interposée à VINEY et PARDE (1960) qui, à l'instar de certains forestiers allemands, préconisaient des éclaircies plus prudentes avec, semble-t-il, comme objectif des cernes inférieurs à $1,5 \mathrm{~mm}$, et qui concluaient que la sylviculture đu chêne rouvre était un art délicat dont le succès était «conditionné par une forêt longtemps dense ».

Au cours de la dernière décennie, une plus grande attention portée aux prix de revient des dégagements de semis et des premières éclaircies, jointe à la raréfaction de la main-d'cuvre forestière, ont conduit à penser que ces opérations onéreuses pourraient avantageusement être concentrées sur les individus susceptibles de former le peuplement définitif dont l'espacement peut facilement être déterminé à partir de la taille maximale des houppiers dans la station considérée; tel était le but de la pré-désignation des arbres de place dont VENET (1967 a et 1968) s'est fait l'ardent protagoniste, ou de l'individualisation précoce des arbres d'avenir, chère à de ST VAULRY (1969).

Or il est clair qu'en attirant matériellement l'attention sur un certain nombre de tiges et en faisant à leur profit toutes les opérations culturales, on risque, que cela soit ou non le but recherché, de favoriser leur croissance, done de leur faire produire des cernes plus larges dans des limites il est vrai imposées par la fertilité de la station.

Quant aux aspects purement technologiques de la largeur des accroissements, MAROTTE (1956) indiquait comme qualité essentielle du chêne de tranchage, des accroissements serrés et réguliers sur le modèle de Tronçais et Bercé ayant « moins de $1 \mathrm{~mm}$ par accroissement annuel $n$.

VENET (1967 b), sans prendre parti sur le fond, mentionnait, sur la base de I pour le chêne de taillis sous futaie, des prix moyens de 1,5 pour le chêne de futaie à cernes de 2 à $3 \mathrm{~mm}$, de 2,5 pour ceux à cernes de 1,4 à $2 \mathrm{~mm}$, et une fourchette de 4 à 8 selon les provenances pour les arbres à cernes de moins de $1 \mathrm{~mm}$.

Les forestiers allemands, auxquels l'importance du problème n'a pas non plus échappé, semblent avoir à ce sujet des avis assez divergents puisque VANSELow (1956) donne comme largeur de cernes la plus économiquement souhaitable 1,2 à $1,5 \mathrm{~mm}$, alors que SchuLz (1959) affirme que la largeur des accroissements ne joue pas le rôle absolument déterminant que beaucoup lui attribuent, et que le prix du bois de tranchage est plus en relation avec sa couleur qu'avec l'épaisseur des cernes annuels.

Au reste, quelles que soient leurs divergences sur le problème de la largeur des accroissements, tous les auteurs mentionnés ci-dessus sont unanimes pour reconnaitre que, toutes choses égales par ailleurs, le prix des grumes de tranchage augmente avec le diamètre quí, à durée de révolution égale, est bien entendu en liaison fonctionnelle avec l'épaisseur des couches d'accroissements.

Si l'on veut sortir des discussions théoriques souvent basées sur des idées reçues ou 
même sur des considérations d'ordre affectif, la nécessité apparait d'étudier de façon approfondie les relations entre la vitesse de croissance et les diverses caractéristiques physiques ou mécaniques du bois de chêne.

Tel est le but du présent travail consacré à une scule forêt, combien prestigieuse, celle de Tronçais, où ces liaisons sont analysées à trois niveaux : à l'intérieur de diverses parcelles unitaires, entre moyennes de parcelles, et enfin à l'intérieur des arbres dans le cas où des accẻlérations de croissance, voulues ou non, ont été constatées.

\section{I. - MATÉRIEL ET MÉTHODE}

Le matériel utilisé se divise en deux catégories : I'une constituée d'échantillons non destructifs (carottes de sondage de $5 \mathrm{~mm}$ de diamétre) prélevés sur un grand nombre d'arbres mais ne permettant que l'étude d'un nombre limité de caractéristiques, l'autre formée d'éprouvettes normalisées obtenues après abattage de quelques arbres représentatifs sur lesquelles ont été analysées les liaisons entre les critères mesurés sur carottes de sondage et ceux qui ne pouvaient pas l'être.

\section{1. - Essais non destructifs}

\subsection{Echantillsnnage au niveau " parcelle $n$.}

5 parcelles ont été étudiées, situées dans 5 séries différentes correspondant à des conditions de stations et de traitements variées :

La parcelle 69 de $1^{\text {re }}$ série est une jeune futaie âgée de 70 a 90 ans; le sol est un sol brun acide sur grès du trias, faiblement lessivé.

La parcelle 22 de $2^{c}$ série était à l'état de coupe définitive lors de l'expérience; elle repose sur un sol brun lessivé à pseudogley sur grès du trias.

La parcelle 61 de $3^{e}$ série porte une très vieille futaie de 300 ans en cours de coupe définitive; elle est située sur une zone de transition entre un sol brun faiblement lessivé sur sables et argiles miopliocènes et un sol brun acide sur grès du trias.

La parcelle 35 de 5 esérie porte une futaie de 160 ans à l'état de dernière coupe secondaire; le sol est un sol lessivé à pseudogley sur sables et argiles mio-pliocènes.

La parcelle 17 de $6^{\mathrm{e}}$ série est également à l'état de dernière coupe secondaire, mais plus âgée que la précédente ( 175 ans environ); le sol est très voisin de celui de la parcelle 35 de $5^{\circ}$ série.

\subsection{2. Échantillonnage au niveau « arbre $\$$.}

Dans chacune des 5 parcelles retenues ont été sondés 25 arbres pris au hasard à l'intérieur du peuplement, mais en éliminant les plus beaux individus, de façon que les trous de sondage ne risquent pas de provoquer une dépréciation des meilleures billes de tranchage; pour éviter un biais possible dans l'expérimentation, les prélèvements ont été effectués sur des sujets présentant certains défauts indépendants de la qualité intrinsèque du bois : pourritures descendantes ou présence d'une fourche à un niveau tel qu'elle ne puisse interférer avec les caractéristiques mesurées sur carottes à hauteur d'homme, ...

Un seul prélèvement a étẻ fait à environ $1,30 \mathrm{~m}$ de hauteur en éliminant les zones de bois de tension ou celles comportant des cannelures trop prononcées.

La longueur des échantillons recueillis était de l'ordre de $15 \mathrm{~cm}$, ce qui permettait dans les 4 parcelles de haute futaic d'avoir une bonne représentation de la qualité du bois adulte dans la partie effectivement susceptible d'étre utilisée comme bois de tranchage, alors que dans la parcelle 69 de $1^{\text {re }}$ série, on disposait d'échantillons allant de la moelle jusqu'à l'écorce et s'étendant done à la fois sur du bois juvenile et du bois adulte.

Sur les carottes entières a été déterminée l'infradensité globale par la méthode de saturation intégrale (POLGE, 1963); les retraits tangentiels et axiaux entre l'état saturé et l'état stabilisé à $15 \%$ d'humidité ont été mesurés à l'aide d'un capteur de déplacement à transformateur différentiel suivant une 
méthode décrite par ailleurs (Potge et al., 1973); le retrait radial a été apprécié dans les mêmes conditions à l'aide d'un micromètre au $1 / 100^{\circ}$ de $\mathrm{mm}$; le retrait volumétrique a ćté calculé par défaut en additionnant les trois retraits précédents, c'est-ai-dire en négligeant les termes de $2^{\mathrm{c}}$ et $3^{\circ}$ ordre.

Tous les échantillons ont été radiographiés parallèlement au fil du bois de façon à permettre des dénombrements et des mesures de largeurs d'accroissements, ainsi que des observations sur la structure du bois, mais de telles radiographies axiales ne peuvent être utilisées pour l'analyse densitométrique du bois dans le cas particulier des feuillus a zone poreuse, et ce pour plusieurs raisons :

Le diamètre des vaisseaux est du mème ordre de grandeur que la hauteur du faisceau analyseur du microdensitométre, en sorte que le profil obtenu varie suivant que l'axe d'exploration passe ou ne passe pas par un vaisseau.

Les rayons ligneux, qui correspondent à des zones à forte densité, ne sont qu'exceptionnellement parallèles à l'axe de la carotte; là également, les résultats sont différents suivant que le microdensitomètre explore ou non l'image d'un rayon ligneux.

Enfin, les plages de fibres elles-mêmes sont parfois de petite étendue, et leur répartition présente un certain caractère aléatoire.

Pour toutes ces raisons, il a été jugé préférable de radiographier á nouveau les carottes de sondage en utilisant un rayonnement tangentiel, de façon que l'image obtenue donne une meilleure représentation de la structure du bois en intégrant tous les éléments anatomiques rencontrés par le rayonnement dans l'épaisseur de $5 \mathrm{~mm}$ traversée.

L'inconvénient des radiographies tangentielles est qu'elles risquent de donner par endroits des images floues lorsque tous les accroissements ne sont pas paralleles les uns aux autres, ou encore lorsque leur rayon de courbure est trop important: I'analyse densitométrique a été faite en conséquence sur une longueur de radiographie d'environ $3 \mathrm{~cm}$ représentant en moyenne 25 accroissements annuels (les valeurs extrèmes étaient de 9 et de 51), sélectionnée en fonction de la seule netteté de l'image des cernes; cette façon de procéder n'a introduit aucun biais dans l'expérience, car il s'agit là d'un facteur qui est tout à fait indépendant de la qualitè du bois; ce fragment correspondait le plus souvent aux derniers centimètres et même, lorsque ce n'était pas le cas, la zone étudiée était choisie de manière à présenter des caractéristiques comparables à la zone finale, en particulier au point de vue largeur des aceroissements.

La radiographie tangentielle a été faite en utilisant les caractéristiques d'exposition suivantes :

- distance source-film : $2,50 \mathrm{~m}$;

- intensité du flux électronique : $20 \mathrm{~mA}$ :

- tension accélératrice : $15 \mathrm{kV}$;

- temps d'exposition : 4 minutes.

Pour l'analyse au microdensitomètre, on a employê un objectif de 4 et us faisceau d'exploration de $0,6 \mathrm{~mm}$ dans le sens axial et de 100 microns dans le sens radial; sur les enregistrements obtenus. ont été mesurées au coordinatographe les densités maximales et les densités minimales annuelles; enfin, le fragment de carotte ayant servi à l'analyse microdensitométrique a été découpż et son infradensité a été mesurée comme celle de l'ensemble de la carotte par la méthode de saturation intégraie.

\section{2. - Essais destructifs}

8 billons au total provenant de 8 arbres différents ont servi à confectionner 548 éprouvettes normalisées destinées à des essais en flexion dynamique, flexion statique, compression axiale, de dureté, à des mesures de retrait ou à des études microdensitométriques; 4 arbres ( 2 de la parcelle 22 de $2^{\circ}$ série et 2 de la parcelle 35 de $5^{c}$ ) avaient avant abattage fait l'objet de prélevements non destructifs; les quatre autres provenaient d'autres parcelles, a savoir pour trois d'entre eux de la parcelle 25 de $2^{\circ}$ série et le troisième de la parcelle 61 de $3^{\circ}$ série.

\subsection{Essais mécaniquers.}

Les éprouvettes se répartissent en deux types :

- type I : éprouvettes destinées aux essais en flexion dynamique; ces barreaux parallélépipédiques de $2 \mathrm{~cm} \times 2 \mathrm{~cm} \times 30 \mathrm{~cm}$ sont placés contre deux appuis distants de $24 \mathrm{~cm}$ et brisés par l'action d'un mouton-pendule dont la puissance, au moment du choc, s'éléve ả $10 \mathrm{kgm}$ (NF B 51-009). L'effort est dirigé dans le sens tangentiel du bois; les éprouvettes de type I n'ont subi que cet essai.

- type II : éprouvettes destinées aux essais en flexion statique et en compression axiale, à la détermination de la dureté et du retrait entre l'état saturé et l'état sec à l'air. 
Les èprouvettes de flexion statique ont les mėmes dimensions que celles qui sont destinées aux essais en flexion dynamique. Elles sont posées sur deux appuis distants de $28 \mathrm{~cm}$. L'effort s'exerce tangentiellement au milieu de la portée par l'intermédiaire d'un couteau cylindrique (NF B 51-008). Cet essai permet la mesure de la fleche à la rupture et de la charge de rupture.

Des parties intactes des éprouvettes ayant subi l'essai précédent sont tirés deux parallélépipèdes de $2 \mathrm{~cm} \times 2 \mathrm{~cm} \times 6 \mathrm{~cm}$ destinés, l'un à l'étude de la résistance en compression axiale, l'autre à la mesure de la dureté.

Dans le cas de la compression axiale, l'effort est dirigé axialement, parallelement au fil du bois (NF B 51-007).

La mesure de la dureté est faite en partie selon la modalité de la norme NF B 51-013; le cylindre d'empreinte exerce tangentiellement pendant 5 secondes une charge de $200 \mathrm{kgf}$ sur une face radiale de l'éprouvette.

Entre le cylindre et le bois est interposé un morceau de papier-carbone qui imprime sur I'échantillon la trace de la surface de contact.

Selon la norme précitée, la détermination de la profondeur d'empreinte a été faite à partir de la mesure sur le bois de la largeur de la marque qu'y laisse le carbone; dans le cas du chêne, cette méthode a donné de meilleurs résultats que la pesée de la surface de papier-carbone portant la marque du bois.

Un cube de $2 \mathrm{~cm}$ de côté est également tiré de chaque éprouvette du type II. II est destiné aux mesures du retrait entre l'état saturé, aprés immersion prolongée dans l'eau, et l'état sec à l'air, après conditionnement à l'étuve hygrométrique. La rétractibilité est déterminée suivant les trois directions du plan ligneux : axiale, radiale et tangentielle.

Enfin, un parallélépipède de $2 \mathrm{~cm} \times 2 \mathrm{~cm} \times 0,5 \mathrm{~cm}$ provenant de chaque éprouvette de flexion statique subit une saturation intégrale pour détermination de l'infradensité suivant la méthode déjà employée pour les carottes de sondage.

\subsection{Mesures densitométriques.}

Les échantillons du type III utilisés ont été débitẻs dans les éprouvettes précédentes de façon que leurs dimensions axiales et tangentielles soient de $5 \mathrm{~mm} \pm 0,01$, leur longueur radiale étant de $2 \mathrm{~cm}$ environ; ils ont été radiographiés tangentiellement (à raison d’une centaine par film $18 \times 24 \mathrm{~cm}$ ) suivant des caractéristiques d'exposition identiques à celles employées pour les carottes de sondage, sauf en ce qui concerne le temps d'exposition qui a été porté à 4' $30^{\prime \prime}$.

Les films, une fois développés, ont été analysés au microdensitomètre enregistreur; sur les graphiques obtenus, on a fait au coordinatographe les mesures suivantes :

- moyennes des densités maximales annuelles,

- moyennes des densités minimales annuelles,

- pourcentage de longueur radiale de densité égale ou supérieure à $500 \mathrm{~g} / \mathrm{dm}^{3}, 600 \mathrm{~g} / \mathrm{dm}^{2}$ et $700 \mathrm{~g} / \mathrm{dm}^{2}$,

- largeur moyenne des accroissements annuels.

Le tableau 1 ci-dessous résume la répartition des éprouvettes des trois types :

TABLEAU 1

\begin{tabular}{c|c|c|c|c}
\hline \hline \multirow{2}{*}{ Numéro du billon } & Origine du billon & Type I & Type II & Type III \\
\cline { 2 - 5 } 1 & Parcelle 25 de 2e série & 2 & 4 & - \\
2 & - & 3 & 17 & - \\
3 & - & 5 & 15 & 34 \\
84 & Parcelle 35 de 5e série & 50 & 49 & 40 \\
85 & - & 50 & 50 & 33 \\
9 & Parcelle 62 de 3e série & 30 & 6 & 39 \\
104 & Parcelle 22 de 2e série & 50 & 47 & 46 \\
I06 & - & 50 & 50 & 192 \\
TorAL & & 240 & 308 & 46 \\
\hline \hline
\end{tabular}




\section{II. - RÉSULTATS}

\section{1. - Essais non destructifs}

\subsection{Liaisons entre arbres à l'intérieur des parcelles.}

La matrice des coefficients de corrélation correspondante fait l'objet du tableau 2 sur lequel n'ont été reportés, à une seule exception près, que les coefficients significatifs au seuil de $5 \%$ et au-dessous; un trait plus épais sépare les variables mesurées sur des matériels différents : échantillons ou carottes entières. Les liaisons établies de part et d'autre de cette séparation doivent être considérées avec une certaine réserve, mais gardent cependant leur intérêt car l'échantillon est une partie intégrante de la carotte entière. En outre, de nombreuses caractéristiques du bois à un âge donné sont fortement corrélées avec celles que l'on peut relever à un âge différent, ainsi que le montre par exemple la liaison trés étroite qui existe entre l'infradensité de la carotte et celle de l'échantillon.

\section{Les principales remarques qu'appelle le tableau 2 sont les suivantes :}

La densité minimale annuelle est liée positivement à son écart-type (qui traduit ses variations dans le temps à l'intérieur d'un même individu), alors qu'une liaison de signe contraire (qui n'est, il est vrai, significative qu'au seuil de $10 \%$ peut être observée entre la densité maximale et son ćcart-type; faute de connaître le déterminisme de la production des diverses catégories d'éléments anatomiques du bois de chêne, aucune explication du phénomène n'a pu être proposće, mais il méritait sans doute d'être signalé, car des observations analogues ont pu être faites dans d'autres expériences dont les résultats n'ont pas été publiés jusqu'ici.

L'hétérogénéité (différence entre la moyenne des densités maximales et la moyenne des densités minimales annuelles) est très fortement liée de façon positive à la densité maximale. mais l'est beaucoup moins en sens contraire à la densité minimale; en fait, le rèsultat apparait encore plus étonnant lorsque l'on considère les liaisons à l'intérieur des diverses parcelles prises isolément (dont les matrices de corrélation ne sont pas données ici dans un but de simplification), puisqu'il y a indépendance complète entre l'hétẻrogénéité et la densité minimale dans la parcelle 61 de $3^{e}$ séric, et que les liaisons entre ces deux caractéristiques ne sont significatives, ni à l'intérieur de la parcelle 35 de $5^{\circ}$ série, ni à l'intérieur de la parcelle 22 de $2^{e}$ série.

Lorsque la largeur des accroissements annuels augmente, on constate une aggravation de l'hétérogénéité due principalement à une élévation des densités maximales annuelles, et ceci ne saurait surprendre; mais ce phénomène est accompagné d'une diminution de l'écarttype des densités maximales; ainsi, les individus à accroissements fins, dont on peut penser qu'ils correspondent aux moins favorisés du point de vue des conditions de nutrition ou de concurrence, réagiraient davantage aux variations annuelles du climat que les arbres qui bénéficient de conditions de végétation plus satisfaisantes.

Aussi bien pour les carottes que pour les échantillons, l'infradensité est, de façon très normale, liée aux différentes composantes de la densité; ce bon recoupement entre des critères mesurés par des méthodes tout a fait indépendantes l'une de l'autre est intéressant à noter.

Les divers retraits sont corrélés positivement avec la densité du bois ou avec certaines de 
TABLEAU 2

Matrice des coefficients de corrélation intraparcelle

Correlation coefficients within plots

Les limites de signification sont de 0,179 au seuil de $5 \%$

$$
\text { - } \quad \text { - } \quad \text { de } 0,234 \text { au seuil de } 1 \%
$$

- 5 - 5 - de 0,295 au seuil de $1 \%$

Significant at $5 \%$ confidence level $r=0,179$

$$
\begin{aligned}
& 1 \% \quad-\quad-r=0,234 \\
& -\quad 1 \% \quad-r=0,295
\end{aligned}
$$

chantillon : sample

Carotte : increment core

Infradensité : specific gravity

Densité maximale : maximal densiry

Densité minimale : minimal density

Ecart-type : sfandard error

Hétérogénéité : heferogeneity

Largeur de cernes : ringwidth

Retrait radial : radial shrinkage

Retrait tangentiel : rangential shrinkage

\begin{tabular}{|c|c|c|c|c|c|c|c|c|c|c|c|c|}
\hline & $\mathrm{dm}$ & $\sigma_{\mathrm{dm}}$ & DM & $\sigma_{D M}$ & DM-dm & $\begin{array}{l}\text { Largeur } \\
\text { cernes }\end{array}$ & $\begin{array}{c}\mathrm{PO} / \mathrm{VS} \\
\text { échantillon }\end{array}$ & $\begin{array}{l}\text { PO VS } \\
\text { carotte }\end{array}$ & RT & RR & RA & RV \\
\hline Densitê minimale (dm) & I & & & & & & & & & & & \\
\hline Ecart-type densité minimale $\left(\sigma_{\text {dm }}\right)$ & 0,342 & 1 & & & & & & & & & & \\
\hline Densité maximale (DM) & 0,484 & 0,254 & 1 & & & & & & & & & \\
\hline Écart-type densité maximale (Gıu) & 1 & 1 & $-0,150$ & 1 & & & & & & & & \\
\hline Hètèrogènèité (DM - dm) & $-0,342$ & 1 & 0,656 & 1 & 1 & & & & & & & \\
\hline Largeur moyenne des cernes & 1 & 1 & 0,210 & $-0,360$ & 0,254 & 1 & & & & & & \\
\hline Infradensité échantillon (PO/VS) & 0,568 & 0,190 & 0,757 & 1 & 0,324 & 0,202 & 1 & & & & & \\
\hline Infradensité carotte (PO/VS) & 0.586 & 0.291 & 0,740 & 1 & 0,288 & 1 & 0,803 & 1 & & & & \\
\hline Retrait tangentiel (RT) & 0,250 & 0,189 & 1 & 1 & 1 & l & 0,259 & 0.295 & 1 & & & \\
\hline Retrait radial ( $R R$ ) & 0.192 & l & 1 & 1 & 1 & $-0,233$ & 1 & 0,224 & 1 & 1 & & \\
\hline Retrait axial (RA) & I & 1 & I & 1 & 0,188 & 1 & 1 & 1 & 0,223 & 1 & 1 & \\
\hline Retrait volumètrique ( $R V$ ) & 0,261 & 0,214 & 0,213 & 1 & 1 & l & 0,273 & 0,361 & 0,885 & 0,419 & 0.489 & 1 \\
\hline
\end{tabular}

Retrait axial : axial shrinkage

Retrait volumetrique : volumetric shrinkage 
ses composantes, ce qui confirme des liaisons bien admises pour l'ensemble des espéces ligneuses; seule surprend la corrélation négative entre la largeur des accroissements et le retrait radial, mais il convient d'observer que cette liaison n'est significative dans aucune des parcelles en cause et qu'elle est même positive dans deux d'entre elles.

Cas particulier des liaisons entre la densité du bois et la largeur des accroissements. - Ce sont, et de très loin, les plus importantes pour le sylviculteur, puisque la largeur des cernes est un facteur primordial de la production et que la densité du bois est le critère technologique numéro un, en raison des liaisons qui l'unissent à la plupart des autres.

Contrairement à ce qu'on aurait pu penser au vu de la littérature, le coefficient de corrélation intraparcelles correspondant est faible $(r-0,202)$, significatif au seuil de $5 \%$ seulement; qui plus est, aucune corrélation significative n'existe à l'intérieur des parcelles prises isolément, ainsi que cela apparaît très nettement sur la figure 1 qui concerne la parcelle 35 de $5^{e}$ série $(r=0,238 \mathrm{NS})$, la parcelle 31 de $3^{e}$ série $(r=0,054 \mathrm{NS})$, la parcelle 69 de $1^{\text {re }}$ série $(r=0,259 \mathrm{NS})$ et sur la figure 2 relative à la parcelle 17 de $6^{\circ}$ série $(r=0,309$ NS) et la parcelle 22 de $2^{\circ}$ série $(r=0,109$ NS).

On peut voir que dans toutes les parcelles, il est possible de rencontrer des arbres à accroissements relativement larges, qui ne soient cependant pas entachés d'une densité trop élevée; le cas le plus typique est celui de l'arbre n ${ }^{\circ} 20$ qui est, dans la parcelle 61 , celui dont la croissance est la plus rapide et dont cependant la densité du bois est particulièrement faible.

Cette quasi-indépendance entre infradensité et largeur de cernes dans des comparaisons faites entre arbres à l'intérieur d'une même parcelle, ainsi que la très large variabilité de la structure anatomique du chêne en forêt de Tronçais sont illustrées par la figure 3 qui regroupe des négatifs de radiographie de 6 carottes de sondage extraites dans l'ordre de 4 arbres de la parcelle 61 de $3^{e}$ série, d'un arbre de la parcelle 35 de $5^{\circ}$ série et d'un arbre de la parcelle 22 de $2^{e}$ série; sur ces images, les parties denses du bois (rayons ligneux et plages de fibres) apparaissent en noir, alors que les moins denses (zones de petits vaisseaux et de parenchyme périvasculaire) se traduisent par des tons gris plus ou moins foncés, les gros vaisseaux de la zone initiale étant quant à eux représentés par des points blancs de tailles variées.

L'arbre 7 représente le "Tronçais " classique avec des accroissements inférieurs au millimètre; ce n'est cependant pas le moins dense $\left(D=376 \mathrm{~g} / \mathrm{dm}^{3}\right)$ car ses vaisseaux de la zone initiale sont de très petit diamètre.

L'arbre 12 a une densité qui n'est guère plus élevẻe : $402 \mathrm{~g} / \mathrm{dm}^{3}$ (bien que ses cernes soient plus larges) grâce essentiellement à des diamètres de vaisseaux bien supérieurs.

Des quatre arbres de la parcelle 61 , c'est le $\mathrm{n}^{\circ} 20$ qui a le bois le plus léger et le plus tendre ( $D=367 \mathrm{~g} / \mathrm{dm}^{3}$ ), alors que sa croissance est trois fois et demi plus rapide que celle de l'arbre 7; la raison en est que sa zone de bois initial est exceptionnellement développée et formée de vaisseaux de gros diamètre et que dans le bois final les petits vaisseaux et le parenchyme périvasculaire sont beaucoup plus étendus que les fibres.

La densité du bois de l'arbre 21, qui bénéficie lui aussi d'accroissements très satisfaisants, est également très faible $\left(396 \mathrm{~g} / \mathrm{dm}^{3}\right)$; ceci s'explique par l'absence à peu près complète de fibres qui caractérise son plan ligneux.

L'image de l'arbre 98 est donnée parce qu'il représente le cas, qui est censé être le plus 


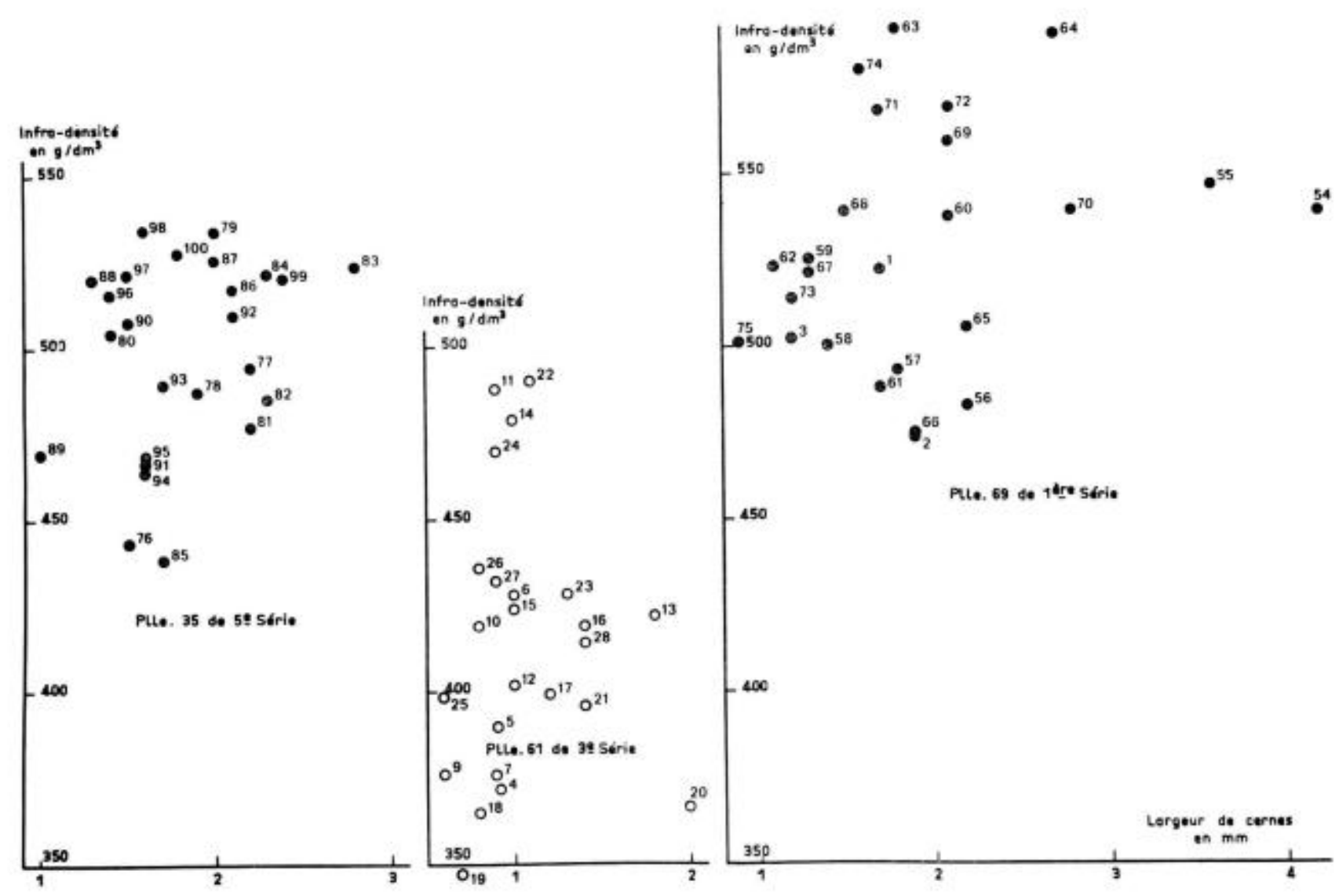

FIG. 1. - Relations entre densité et largear d'accroissements à l'intérieur des parcelles

Fu. 1. - Relationship between specific gravity and ringwidth within plots 


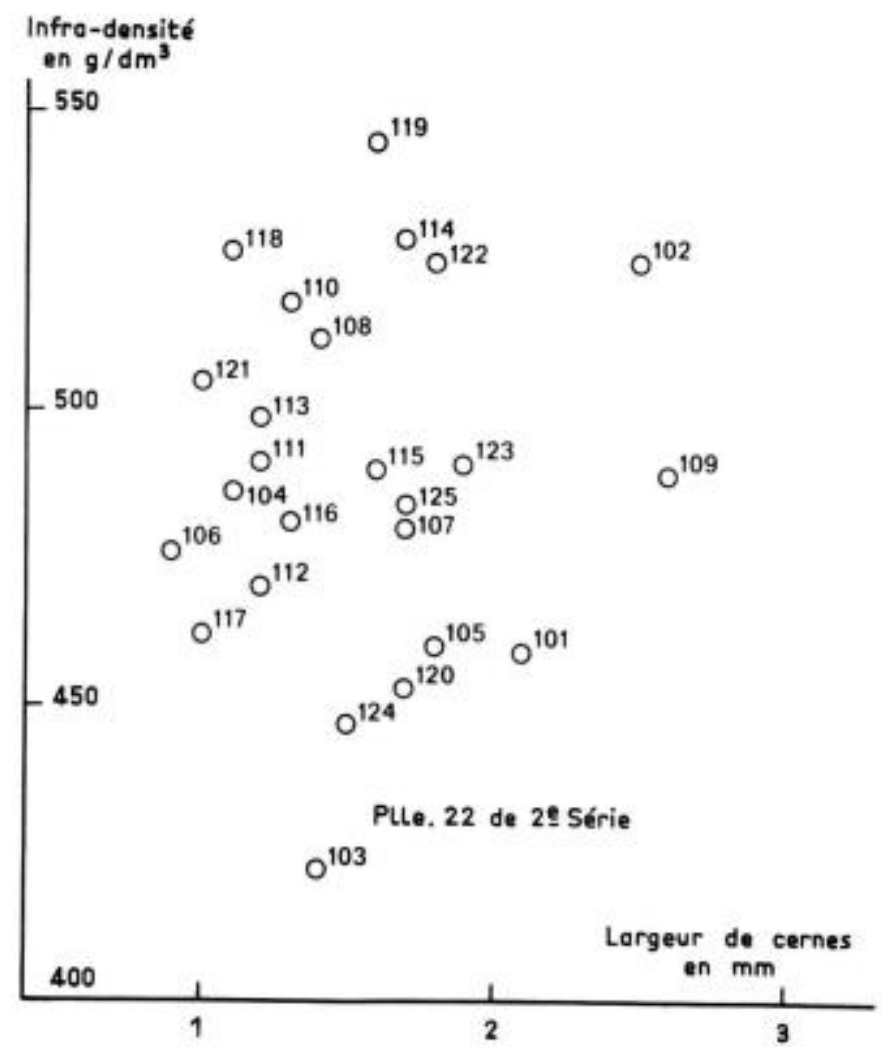

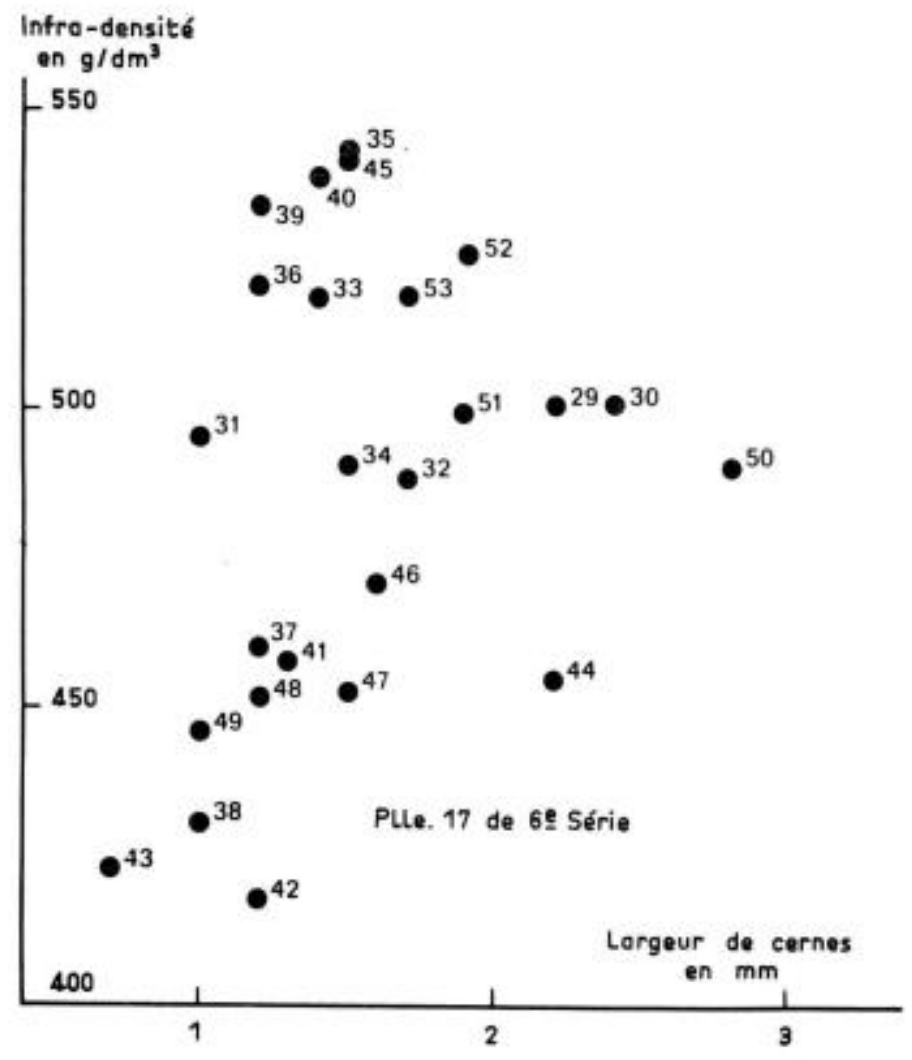

Infro-densité

on $g / \mathrm{dm}^{3}$

$$
\bullet_{39} \bullet_{40}^{\mathbf{e}_{45}^{35}}
$$$$
\bullet^{36} \bullet^{33} \bullet^{52}
$$

Infro-densité

en $\mathrm{g} / \mathrm{dm}^{3}$

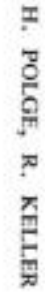

Fig. 2. - Relations enfre densité et largeur d'accroissements à l'intérieur des parcelles

Fig. 2. - Relationship between specific gravity and ringwidth within plots 

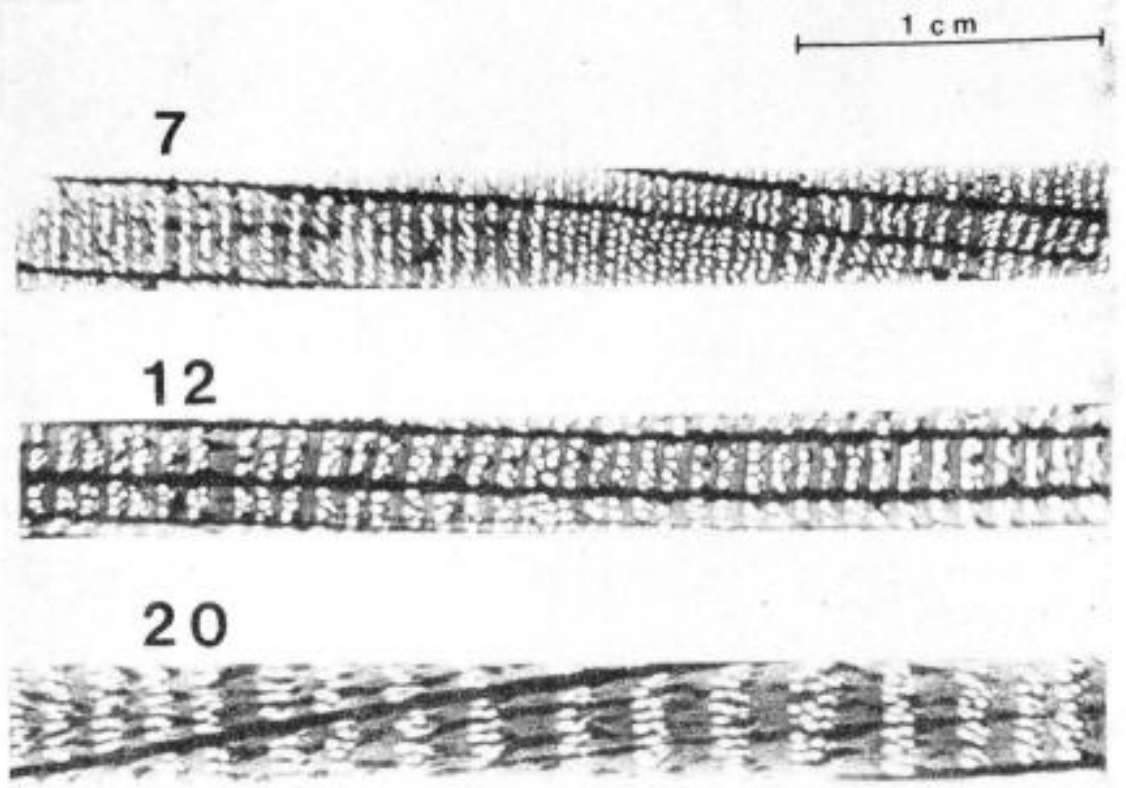

\section{1}

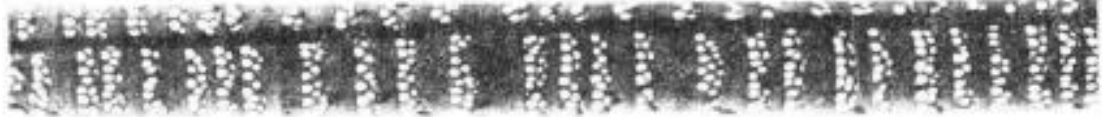

\section{8}

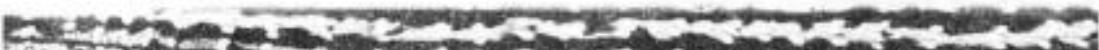

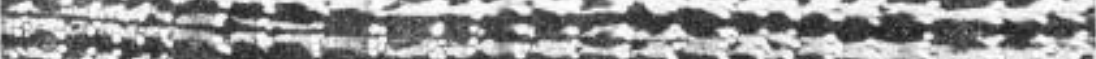

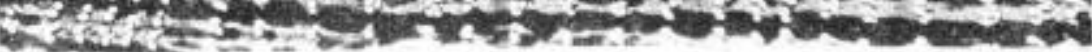

\section{5}

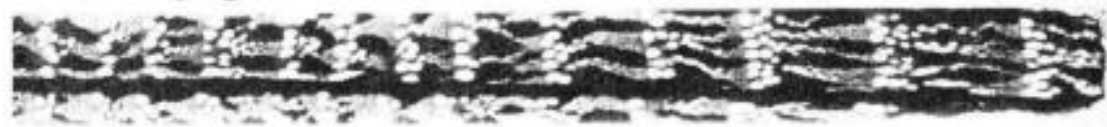

Fic. 3. - Positifs de radiographie de divers échantillons

FiG. 3. - Some positive prints of radiographies 
classique, du bois de chêne à accroissements larges, mais dont la forte densité $\left(534 \mathrm{~g} / \mathrm{dm}^{3}\right)$ tient à une grande abondance des fibres dans le bois final en même temps qu'au faible diamètre des vaisseaux de la zone poreuse.

Tout autre est le cas de l'arbre 105 qui bénéficie d'une croissance particulièrement rapide, mais dont le bois est cependant d'assez bonne qualité ( $\left.D=460 \mathrm{~g} / \mathrm{dm}^{3}\right)$, grâce notamment au fait que des vaisseaux de gros diamètre se retrouvent dans une grande partie de l'accroissement annuel.

Ces quelques exemples montrent que les renseignements tirés de l'examen radiographique recoupent bien ceux qui résultent d'études anatomiques plus fines (VENET, 1973); des uns et des autres, on peut conclure que pour le chêne le problème đes relations entre largeurs des cernes et qualité du bois n'est pas simple, et qu'il convient done de se garder, en la matière, de toute généralisation hâtive.

\subsection{Liaisons entre moyennes de parcelles.}

La matrice des coefficients de corrélation correspondante fait l'objet du tableau 3 ; le nombre réduit de parcelles, qui ne laisse que 3 degrés de liberté pour les coefficients de corrélation, impose une interprétation prudente des résultats; cependant quelques informations intéressantes peuvent en être tirées :

Comme pour les corrélations intraparcelles, l'hétérogénéité est essentiellement liée à la densité maximale, la liaison avec la densité minimale devenant maintenant positive (mais non significative).

De mềme, l'infradensité, aussi bien de la carotte entière que de l'échantillon réduit, est en liaison quasi fonctionnelle avec la densité maximale annuelle, et n'est pas corrélée significativement avec la densité minimale annuelle.

Une certaine interdépendance apparaît entre les différents retraits, notamment, comme c'était le cas au niveau intraparcelles, entre les retraits tangentiels, axiaux et volumétriques. Aucune explication valable ne peut ètre proposée en l'état actuel des connaissances pour rendre compte du fait que le retrait volumétrique diminue lorsque l'écart-type des densités maximales augmente.

Comme précédemment, la liaison la plus intéressante à analyser est celle qui existe entre la largeur des accroissements et l'infradensité du bois; elle est ici positive et très significative; elle apparait clairement sur le graphique de la figure 4 ; ainsi, alors qu'il y a à peu près indépendance entre densité et vigueur de croissance pour les individus d'une même parcelle, on observe au contraire une relation très étroite entre elles lorsque l'on prend en considération les valeurs moyennes de chaque parcelle; la raison en est sans doute qu'à l'intérieur d'un groupe d'arbres donné poussant dans des conditions de sol et de concurrence sensiblement identiques, une large variabilitể existe qui fait que les divers individus réagissent à ces conditions par des comportements physiologiques différents qui entraînent à leur tour de très larges variations de structure du bois.

En revanche, dans des comparaisons entre moyennes de parcelles, le facteur qui parait prépondérant est l'âge du peuplement (il suffit de constater sur la figure 4 que l'échelonnement des points représentatifs se fait de façon très régulière de la parcelle 61 de $3^{e}$ série vieille de 300 ans à la parcelle 69 de $1^{\text {re }}$ série qui n'est âgée que de 80 ans environ, en passant par le 
TABLEAU 3

Matrice des coefficients de correlation interparcelles Correlations coefficients between plots

Les limites de signification sont de 0,878 au seuil de $5 \%$

$$
\text { - } \quad \text { - } \quad \text { - } \quad \text { - de } 0.959 \text { au sevil de } 1 \%
$$

\begin{tabular}{|c|c|c|c|c|c|c|c|c|c|c|c|c|}
\hline & $\mathrm{dm}$ & $\sigma_{\mathrm{dm}}$ & DM & $\sigma_{\mathrm{DM}}$ & DM-dm & $\begin{array}{l}\text { Largeur } \\
\text { cernes }\end{array}$ & $\begin{array}{c}\text { PO/VS } \\
\text { échantillon }\end{array}$ & $\begin{array}{l}\text { PO/VS } \\
\text { carotte }\end{array}$ & RT & RR & RA & RV \\
\hline Densité minimale (dm) & 1 & & & & & & & & & & & \\
\hline Écart-type densité minimale $\left(\sigma_{\mathrm{dm}}\right)$ & l & 1 & & & & & & & & & & \\
\hline Densité maximale (DM) & I & 1 & 1 & & & & & & & & & \\
\hline Écart-type densité maximale $\left(\sigma_{\text {Dw }}\right)$ & 1 & 1 & 1 & 1 & & & & & & & & \\
\hline Hétêrogénéité (DM $-\mathrm{dm}$ ) & 1 & f & 0,973 & l & 1 & & & & & & & \\
\hline Largeur moyenne des cernes & 1 & 1 & 0,972 & 1 & 0,978 & 1 & & & & & & \\
\hline Infradensité échantillon (PO/VS) & I & 1 & 0,998 & 1 & 0,937 & 0.966 & 1 & & & & & \\
\hline Infradensité carotte (PO/VS) & 1 & 1 & 0.996 & I & 0.960 & 0,965 & 0,989 & 1 & & & & \\
\hline Retrait tangenticl (RT) & l & 1 & 1 & f & 1 & 1 & 1 & l & 1 & & & \\
\hline Retrait radial (RR) & 1 & 1 & 1 & 1 & 1 & 1 & 1 & l & 1 & 1 & & \\
\hline Retrait axial (RA) & 1 & 1 & 1 & 1 & 1 & 1 & 1 & t & 0,946 & 1 & 1 & \\
\hline Retrait volumétrique (RV) & 1 & 1 & 1 & $-0,928$ & 1 & 1 & 1 & 1 & 0,937 & 1 & 0,983 & 1 \\
\hline
\end{tabular}


groupe des trois autres parcelles qui portent des peuplements de 160 à 180 ans); le facteur « sol » ne parait, quant à lui, ne pas exercer, au cas particulier, une influence marquée.

En d'autres termes, on note à ce niveau un effet de gradient qui, sauf rares exceptions, se rencontre dans tous les individus et qui fait que chez les feuillus à zone poreuse, la largeur des cernes et la densité du bois diminuent toutes deux parallèlement lorsque l'assise génératrice vieillit.

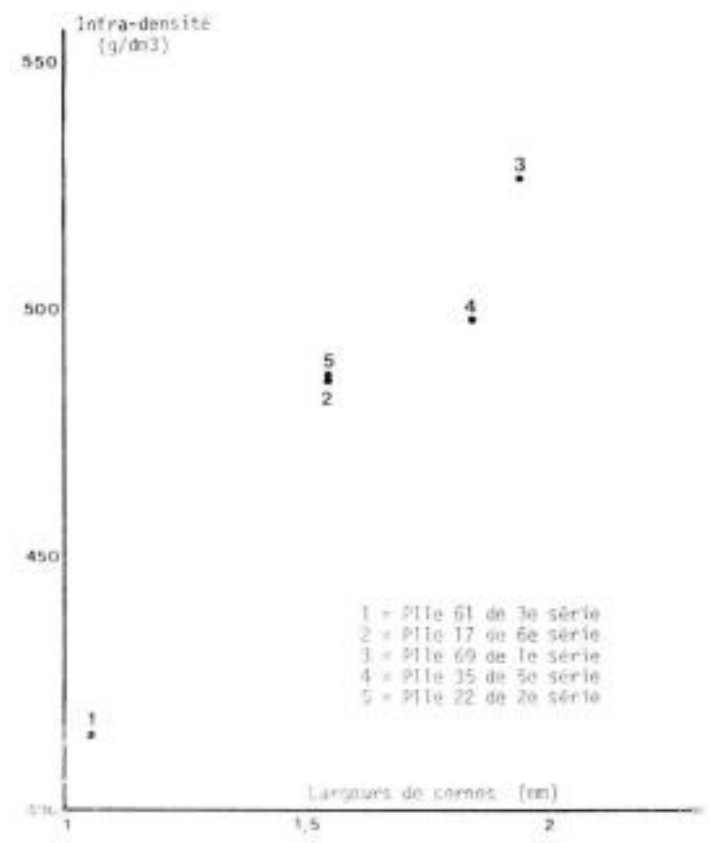

FiG. 4. - Relationx entre densité ef largeur d'accroissement: au nivau des moyennex de parcelles

Fic. 4. - Relationship between spocific gravits and ringwidth between plots

\subsection{Effets d'une accélération de la croissance sur la densité du bois.}

On retrouve ici un problème très important pour le gestionnaire de forêt puisque, partant d'un individu donné dans un environnement donné, on cherche à savoir s'il est possible de lui faire produire des accroissements plus larges sans trop nuire à la qualité du bois; pour répondre à cette question, on a sélectionné, parmi les 125 arbres échantillonnés, tous ceux pour lesquels la radiographie faisait apparaitre une série de cernes larges succédant à une série de cernes étroits; on a isolé les morceaux de carottes correspondants et déterminé leur infradensité; ceci n'a pu être fait que dans les 4 parcelles de haute futaie car dans le jeune peuplement de la parcelle 69 de $1^{\text {re }}$ série, on observe toujours une évolution dans le sens d'une réduction de la largeur des accroissements, et jamais l'inverse.

Au total, 29 individus ont présenté une nette accélération de croissance; sur la figure 5 
sont reportées pour chacun d'eux la densité et la largeur de cernes du fragment à croissance lente et celles du fragment à croissance rapide, en réunissant par un trait les deux points représentatifs d'un même arbre; on voit, qu'à une seule exception près (l'arbre 118), une accélération de la croissance entraîne une augmentation de la densité du bois (qui, pour des utilisations nobles, tels que tranchage ou ébénisterie fine, correspond à une perte de qualité).

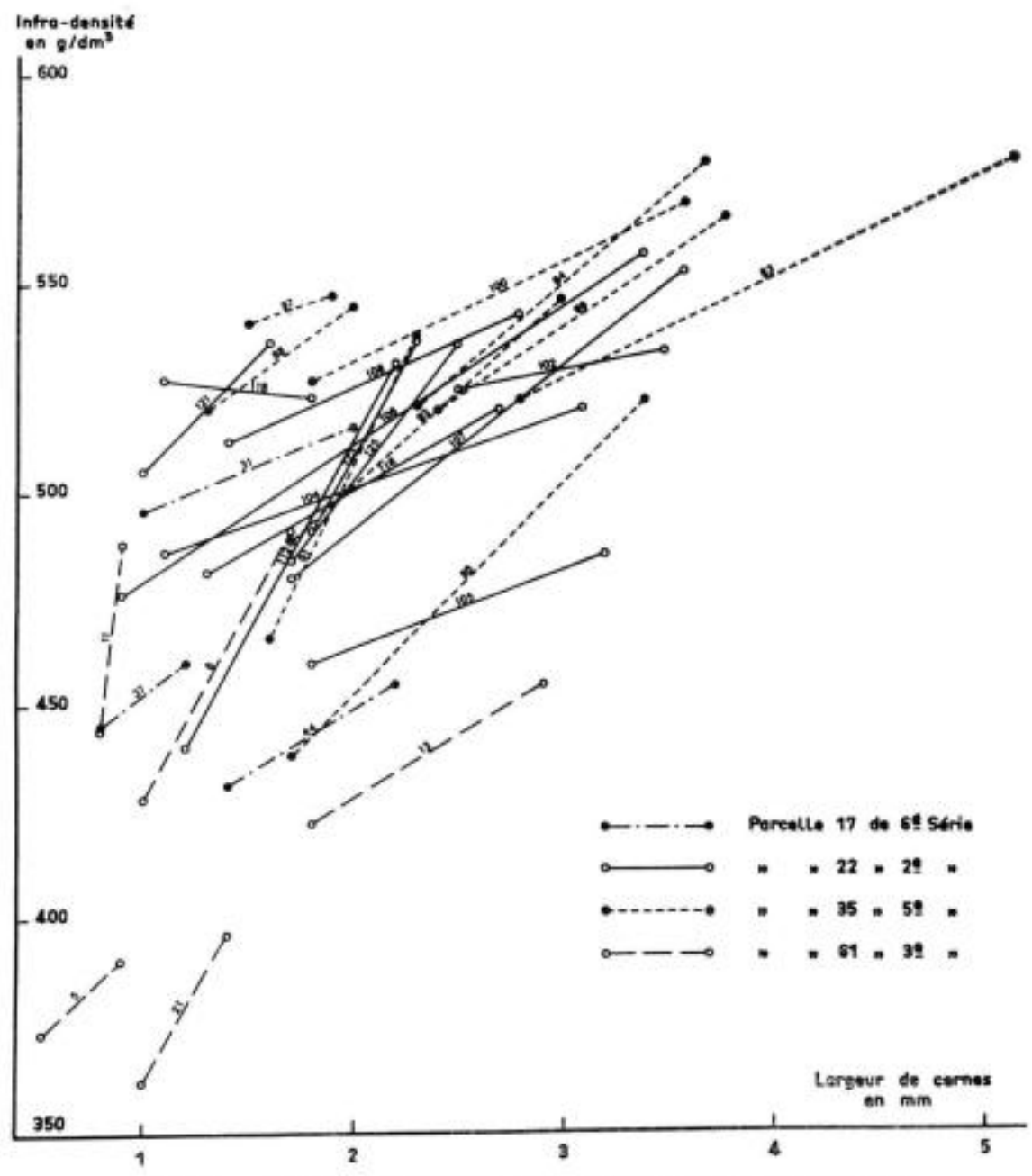

FIG. 5. - Effets d'une accelération de la croissance sur la densité du bois

FiG. 5. - Influence of a growth acceleration on specific gravity

Il apparait en outre que l'aggravation de la densité est d'autant plus forte quéla densité et la largeur moyenne de cernes de l'échantillon sont plus faibles; pour le vérifier, on a reporté sur papier semi-logarithmique (figure 6) les valeurs de la pente des segments de droites de la figure précédente $\left(\frac{\Delta \mathrm{D}}{\Delta \mathrm{L}}\right.$, si $\mathrm{D}$ est l'infradensité et $\mathrm{L}$ la largeur de cernes) en 


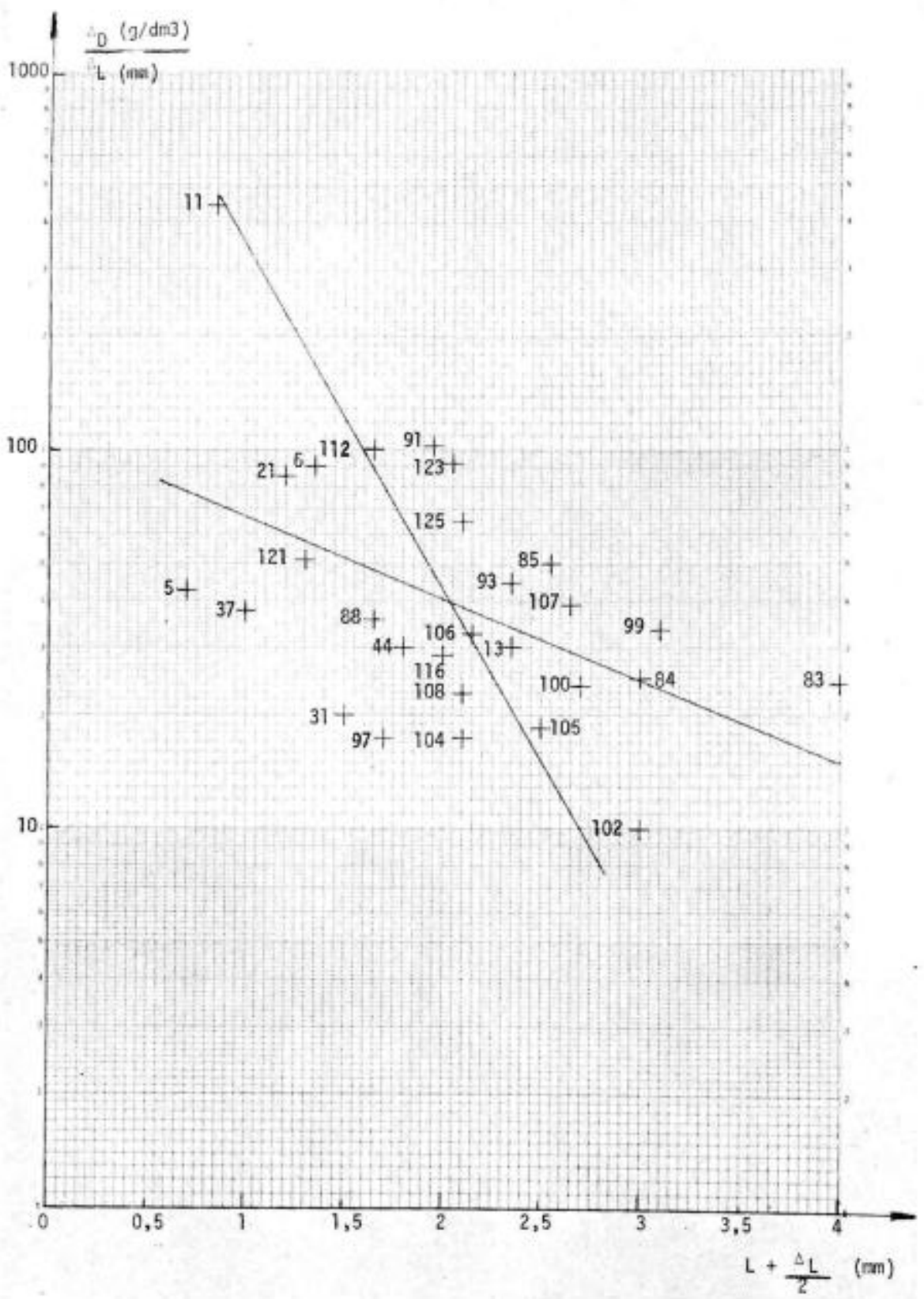

Fig. 6. - Aggravation relative de la densité en fonction de la largeur moyenne des accroissements FiG. 6. - Relative value of the increase in specific gravity related to the ringwidth 
fonction de la largeur moyenne des deux tranches d'accroissements considérées : $L+\frac{\Delta L}{2}$; le graphique montre que l'on a maintenant affaire à une liaison linéaire, et la corrélation obtenue entre les deux variables $\log$ de $\frac{\Delta \mathrm{D}}{\Delta \mathrm{L}}$ et $\mathrm{L}+\frac{\Delta \mathrm{L}}{2}$ se chiffre à $-0,49$ et est significative au seuil de $1 \%$

L'effet très défavorable d'une forte augmentation de la largeur des accroissements apparait très nettement sur les échantillons 36 et 100 dont un positif de radiographie est reproduit en haut de la figure 7.

Dans les deux cas, il s'agissait d'arbres qui, lorsque leur croissance était relativement lente, produisaient un bois d'excellente qualité, dont la zone de bois final, au reste pauvre en fibres, ne représentait parfois que la moitié de l'accroissement total; lorsqu'ils se sont mis à former des accroissements larges, l'épaisseur de la zone initiale n'a que très peu augmenté, cependant que le bois final était essentiellement constitué de plages de fibres.

Pour préciser le phénomène, on a sélectionné des arbres n'ayant pas subi une accélération aussi brutale de la croissance que les précédents, mais comportant des fluctuations étendues de l'épaisseur des couches annuelles avec des changements assez progressifs de largeur dans un sens comme dans un autre, en excluant bien entendu la période juvénile de la vie de l'arbre oủ le gradient négatif de largeur de cernes prédomine.

Sur les arbres retenus, au nombre de 19, la densité du bois a été déterminée cerne par cerne par planimétrie des profils densitométriques, et les coefficients de corrélation entre cette densité moyenne annuelle et la largeur correspondante de l'accroissement ont été calculés; à l'exception de deux coeflicients négatifs et d'un traduisant une liaison très faible, tous les

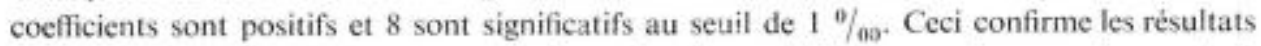
précédents en montrant en outre que, même dans le cas d'alternances de cernes larges et de cernes étroits, la qualité du bois varie, sauf exceptions, en raison inverse de l'épaisseur des accroissements.

Ainsi est clairement mise en évidence par l'expérimentation l'importance du facteur " régularité des accroissements " dont des observations empiriques avaient depuis longtemps conduit sylviculteurs et industriels du bois à soupçonner l'intérêt.

\section{2,14. Autres enseignements tirés des essais non destructifs.}

2.141. Étude d'un arbre ayant poussé à l'état isolé, - En sus des 125 èchantillons en provenance de peuplements forestiers qu'on peut considérer comme normaux pour la forêt de Tronçais, a été prélevée une carotte de sondage dans un arbre de la parcelle 25 de $4^{\circ}$ série qui manifestement a bénéficié toute sa vie d'une absence à peu près totale de concurrence au niveau de la cime vivante, à en juger par le diamètre de son houppier qui est de l'ordre de $20 \mathrm{~m}$.

Bien qu'il faille se garder de généraliser à partir d'un cas isolé, cette réserve, qui a été conservée sur pied lors de la coupe définitive intervenue il y a 20 ans, est intéressante à étudier car elle peut, dans une certaine mesure, préfigurer le comportement d'arbres de futaies qui seraient fortement éclaircis durant toute leur existence. 


\section{6}

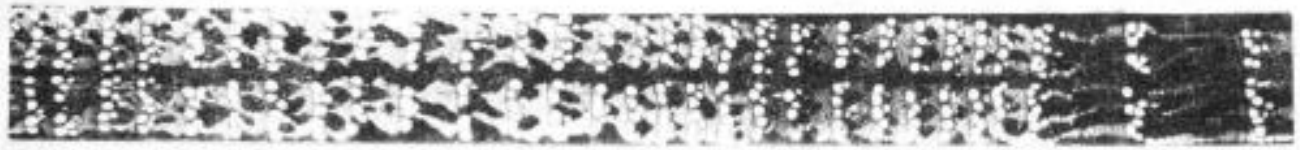

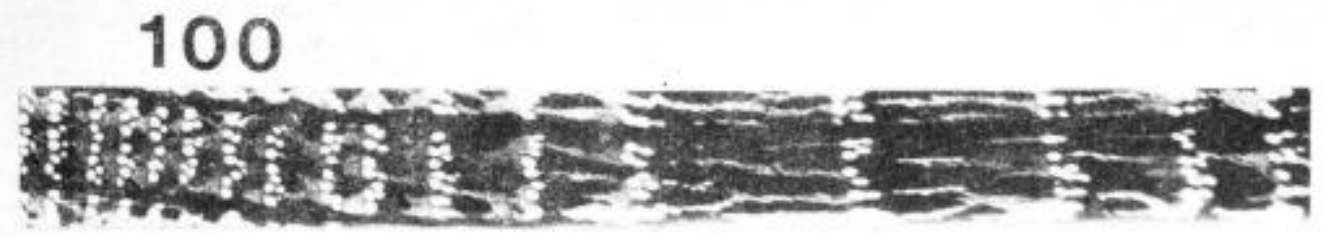

\section{A}

1

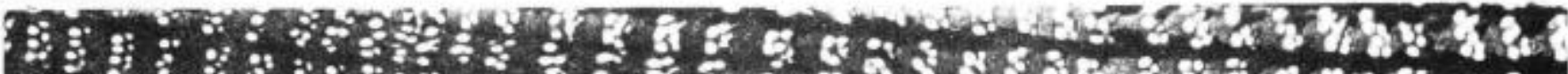

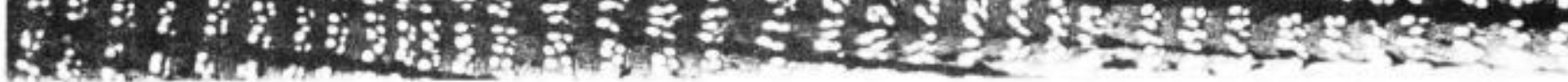

2

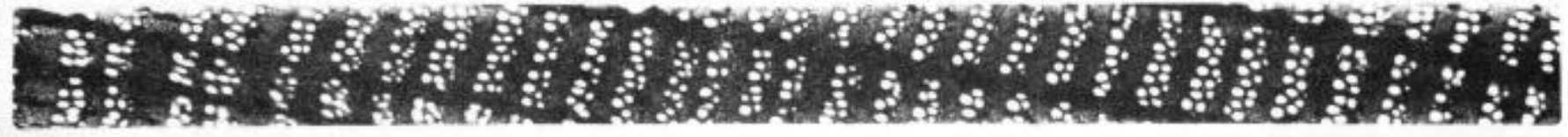

FIG. 7. - Pesitifs de radiographic

- à la partie supérieure : de deux arbres ayant bénéficié d'une forte accellération de la croissance - à la partie infériesare : d'un arbre isolé à houppier tr ès développé

Fic. 7. - X-ray photograph positive prints 
L'image des accroissements formés pendant environ 70 ans est reproduite sous la référence $A_{1}$ et $A_{2}$ en bas de la figure 7 ; on observe une très grande régularité de la largeur des cernes et également une structure très favorable du bois marquée par une zone poreuse très large et une absence à peu près complète de fibres dans le bois final; comme conséquence, la densité est relativement faible $\left(444 \mathrm{~g} / \mathrm{dm}^{3}\right)$ et l'homogénéité très satisfaisante; on a donc indiscutablement affaire à une qualité " tranchage ", mais il faut bien reconnaittre que la largeur des accroissements reste très modeste ( $1,5 \mathrm{~mm}$ environ); tout se passe comme si les arbres de haute futaie ayant longtemps crû à l'état serré et bénéficiant soudain d'une diminution de la concurrence pouvaient, grâce peut-être à une amélioration brutale de leur bilan hydrique (approvisionnement en eau dans le sol beaucoup plus abondant pour une évapotranspiration qui n'augmente que faiblement), se mettre à produire pendant un nombre limité d'années, des cernes de grande largeur; avec un arbre isolé, au contraire, il y aurait toujours un équilibre relativement précaire entre la disponibilité en eau dans le sol et la quantité qui est dissipée dans l'atmosphẻre par un houppier largement développé, ce qui amènerait la formation de cernes réguliers, mais d'une largeur qui demeurerait conditionnée, outre la fertilité de la station, par les ressources en eau.

2.142. Liaisons juvénile-adulte, - 11 est toujours difficile, sur des arbres de fort diamètre, de faire des sondages au cæeur, et même dans le cas de la jeune futaie de la parcelle 69 , on a, compte tenu de la valeur des arbres en cause, hésité à multiplier les prélèvements jusqu'à ce qu'on atteigne la moelle; ceci a empêché de procéder à une analyse statistique des liaisons juvénile-adulte en matière de densité du bois qui eût cependant été souhaitable, mais il n'est guère douteux que ces liaisons soient très fortes, ainsi que le montrent les quatre èchantillons de la figure 8 qui ne correspondent pas à un échantillonnage biaisé, mais sont seulement ceux qui se sont avérés être les plus radiaux.

Bien que les premiers cernes côté coxur soient quelque peu flous pour deux des arbres en cause, il apparaît clairement que les caractéristiques du bois à proximité immédiate de la moelle préfigurent, bien qu'en systèmatiquement moins satisfaisantes, celles que l'on retrouve 50 ans plus tard.

Les arbres 63 et 71 ont dès le début une forte proportion de fibres qui se manifeste également dans les derniers accroissements produits; les premiers cernes de l'arbre 57 se caractérisaient déjà par un bois initial relativement large et par un bois final riche en petits vaisscaux et en parenchyme périvasculaire, ce qui donne une image positive peu foncée, et il en est de même pour l'arbre 65 qui, malgré une zone poreuse moins développée, a cependant donné du bois de bonne qualité dès son jeune àge grâce à une proportion de fibres peu élevée.

2.143. Influence de la tardiveté du débourrement. - Il existe une loi qui parait avoir une portée générale, dans les espèces résineuses tout au moins, qui veut que les individus ou les provenances tardives aient tendance à former du bois de plus faible densité; ceci est vrai lorsque l'on compare entre elles au stade juvénile des provenances d'Abies grandis (POLGE, 1968) ou d'Epicéa commun (LACAZE et POLGe, 1970), et il en est de même entre individus d'une même population d'Epicéa (Thiercelin, 1970) ou de Douglas (résultats non publiés jusqu'ici); il était donc intéressant de voir si une loi analogue existait pour le chêne, ce qui eût permis d'envisager des possibilités de sélection d'individus tardifs à densité faible, c'està-dire combinant deux facteurs favorables. 


\section{7}

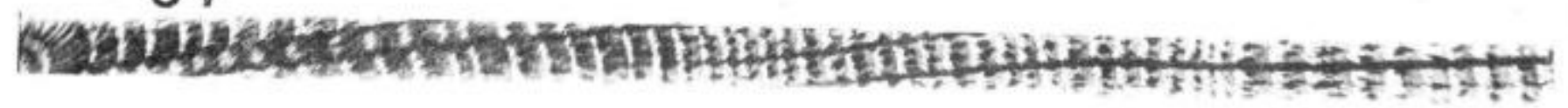

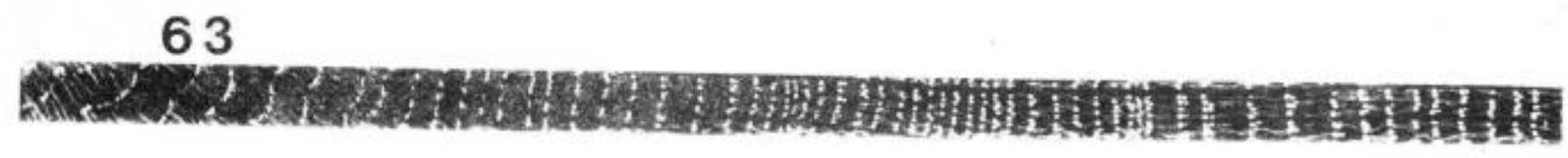

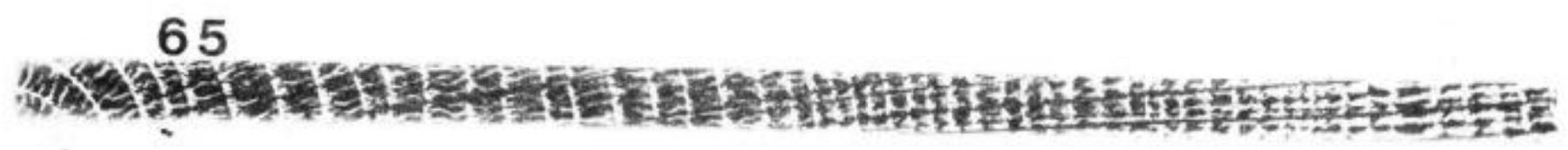

71

$1 \mathrm{~cm}$

Fug. 8. - Positifs de radiographie montrant les laisons juvénile-adulte an poimt de vue structure da bois

Fio. 8. - X-ray photograph positive prints showing the relationship between wood structure at the juvenile and the mature stage 
Pour le vérifier, une reconnaissance supplémentaire a été faite au printemps 1972, au moment où le débourrement présentait le maximum de variabilité individuelle; l'échelle de notation suivante a été utilisée :

1 = début de débourrement;

2 = débourrement du haut en bas de la cime;

$3=$ feuilles en cours de développement;

$4=$ feuilles bien développées du haut en bas.

Des analyses de variance faites en séparant l'effet " parcelle " n'ont malheureusement fait apparaitre aucune différence significative entre les densités moyennes, ou même entre les densités minimales, qui auraient pu être davantage liées à la reprise d'activité végétative au printemps que les précédentes; accessoirement, cette reconnaissance a permis de repérer, parmi tous les échantillons de l'expérience, quatre chênes pédonculès sûrs, mais là également, ils n'ont paru se différencier en rien des chênes rouvres en ce qui concerne la structure et les caractéristiques du bois.

\section{2. - Essais destructifs relatifs aux éprouvettes du type II}

\subsection{Liaisons de T'infradensité avec les propriétés mécaniques et le retrait (tableaux 4 et 5 ).}

L'infradensité est en corrélation positive avec la résistance en flexion statique, la résistance à la compression axiale et la dureté, aussi bien dans la matrice des coefficients de corrélation intrabillons ( 299 degrés de liberté) que dans la matrice interbillons ( 6 degrés de liberté) qui porte sur leurs moyennes.

Forte densité et résistance mécanique élevée tendent donc à aller de pair, à la fois sur l'ensemble des éprouvettes provenant des 8 billons en cause et sur les valeurs moyennes de ceux-ci.

Le retrait radial, qui figure sur les deux tableaux de corrélation, est lié positivement à l'infradensité, aux résistances en flexion statique et en compression axiale, à la dureté et au retrait volumétrique. II n'est en corrélation, ni avec le retrait tangentiel, ni avec le retrait axial.

Le retrait tangentiel présente les mémes relations, mais seulement sur le tableau des coefficients intrabillons.

Le retrait axial $n$ 'a montré qu'une liaison négative $(r=-0,769)$ avec la flèche à la rupture en flexion statique, dans le cas des corrélations interbillons; il ne faut toutefois noter ce résultat qu'avec prudence, étant donné le faible nombre de degrés de liberté dans ce type de calcul,

Le retrait volumétrique est le reflet des liaisons observées pour le retrait radial ou le retrait tangentiel.

En ce qui concerne les relations avec l'infradensité, les chiffres obtenus dans le cas des éprouvettes normalisées concordent bien avec ceux qui figurent sur le tableau 2 relatif aux carottes. 


\section{TABIFAU 4}

Matrice des coelficients de corrélation intrabillons Correlation coefficienrs within bolts

Les limites de signification sont de 0,230 au seuil de $5 \%$

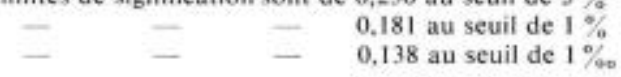

PO/VS - infradensité - specific gravity

RFS = contrainte maximale en flexion statique = maximum load in static bending

FLR = fleche à la rupture en flexion statique maximum deflection in static bending

RCA = contrainte maximale en compression axiale maximum load in compression parallel to grain

DUR - dureté - hardness

HR = humidité de l'échantillon lors des mesures de retrait sample moisture content during shrinkage measurements

HE = humidité de l'échantillon lors des essais mécaniques sample moisture content during mechanical tests

RR - retrait radial - radial shrinkage

$\mathrm{RA}=$ retrait axial $=$ axial shrinkage

RT - retrait tangentiel = tangential shrinkage

RV - retrait volumétrique - volumetric sherinkage

\begin{tabular}{|c|c|c|c|c|c|c|c|c|c|c|c|}
\hline & PO/VS & RFS & FLR & $\mathrm{RCA}$ & DUR & HR & $\mathrm{HE}$ & RR & RA & RT & RV \\
\hline $\mathrm{PO} / \mathrm{VS}$ & 1 & & & & & & & & & & \\
\hline RFS & 0,537 & 1 & & & & & & & & & \\
\hline FLR & 0,201 & 0,568 & 1 & & & & & & & & \\
\hline RCA & 0,656 & 0,608 & 0,265 & 1 & & & & & & & \\
\hline DUR & 0,591 & 0,477 & 0.152 & 0,575 & 1 & & & & & & \\
\hline HR & $-0,139$ & $-0,177$ & I & 1 & 1 & 1 & & & & & \\
\hline $\mathrm{HE}$ & 1 & 0,231 & 0,240 & 1 & 0,172 & 1 & 1 & & & & \\
\hline RR & 0.254 & 0,302 & l & 0,248 & 0.249 & -0.232 & 1 & 1 & & & \\
\hline RA & 1 & I & l & 1 & I & l & $-0,154$ & I & 1 & & \\
\hline RT & 0,338 & 0,220 & 1 & 0,207 & 0,228 & $-0,185$ & 1 & I & 1 & 1 & \\
\hline RV & 0,400 & 0,322 & 1 & 0.291 & 0,305 & $-0,268$ & 1 & 0,637 & 0,197 & 0,843 & 1 \\
\hline
\end{tabular}


TABLEAU 5

Matrice des coefficients de corrélation interbillons

Correlation coefficients between bolts

Les limites de signification sont de 0,707 au seuil de $5 \%$

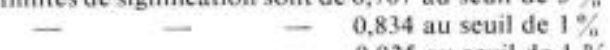

\begin{tabular}{|c|c|c|c|c|c|c|c|c|c|c|c|}
\hline & PO/VS & RFS & FLR & $\mathrm{RCA}$ & DUR & HR & $\mathrm{HE}$ & RR & RA & RT & RV \\
\hline PO/VS & 1 & & & & & & & & & & \\
\hline RFS & 0,779 & 1 & & & & & & & & & \\
\hline FLR & I & 1 & 1 & & & & & & & & \\
\hline $\mathrm{RCA}$ & 0,697 & 0,985 & 1 & 1 & & & & & & & \\
\hline DUR & 0,798 & 0,854 & 1 & 0,859 & 1 & & & & & & \\
\hline HR & 1 & 0.774 & 1 & 0,801 & 1 & 1 & & & & & \\
\hline $\mathrm{HE}$ & 1 & 1 & 1 & $f$ & 1 & 1 & 1 & & & & \\
\hline RR & 0,750 & 0.970 & I & 0,984 & 0,909 & $-0,763$ & 1 & 1 & & & \\
\hline RA & 1 & 1 & $-0,769$ & 1 & 1 & 1 & 0.809 & Y & 1 & & \\
\hline RT & 1 & 1 & 1 & 1 & 1 & 1 & 1 & 1 & 1 & 1 & \\
\hline RV & 0,719 & 0,884 & 1 & 0,886 & 0,788 & f & 1 & 0,868 & 1 & 0,790 & 1 \\
\hline
\end{tabular}

\subsection{Liaisons entre les propriétés mécaniques et le retrait.}

Les corrélations des propriétés mécaniques entre elles ou des retraits entre eux sont généralement élevées.

La dureté présente de forts coefficients de corrélation positive avec la résistance en flexion statique et en compression axiale, la flèche à la rupture, ainsi qu'avec les retraits.

La flèche à la rupture en flexion statique est liée négativement avec le retrait tangentiel et positivement avec le retrait axial, ce qui concorde avec ce que l'on a pu voir à propos de l'infradensitè, qui est corrélée positivement avec le premier, et négativement avec le second.

Toutes les conditions qui tendent à provoquer une augmentation de la densité du bois (en particulier par l'augmentation de la proportion relative des fibres) ont donc une influence favorable sur les propriétés mécaniques de flexion, compression, et pour certains emplois, dureté, mais elles entraînent en revanche une aggravation des retraits radiaux et tangentiels, et la dureté elle-même est un défaut sérieux dans le cas d'une utilisation prévue en tranchage.

Une augmentation de la densité semble concorder avec une diminution du retrait axial, ce qui est favorable et intéressant d'un point de vue théorique, mais néanmoins d'un intérêt pratique réduit, du fait des très faibles valeurs absolues de celui-ci. 


\subsection{Résistance au choc.}

La résistance en flexion dynamique a été mesurée sur les éprouvettes du type I; c'est la seule mesure effectuée sur celles-ci; il existe des différences considérables dans les résultats, dont le plus élevé (billon $\mathrm{n}^{\circ} 2$ ) vaut environ 4.4 fois le plus faible (billon $\mathrm{n}^{\circ} 9$ ).

Des calculs globaux de corrélation ont été faits entre cette caractéristique et les précédentes étudiées aux paragraphes 221 et 222 , et mesurées sur d autres éprouvettes. Comme il s'agit de calculs sur les moyennes, le nombre de degrés de liberté est faible; cependant, un certain nombre de coefficients de corrélation sont significatifs, comme on peut le voir sur le tableau 6 oủ la résistance au choc est liée à l'infradensité, à la résistance en flexion statique et en compression axiale, ainsi qu'aux retraits radiaux et tangentiels.

Il est possible que cette résistance en flexion dynamique soit le reflet des conditions de milieu et de l'âge des arbres d'oủ sont issus les billons étudiés, car elle est réputée liée à l'état et à la constitution des membranes cellulaires et sensible aux facteurs qui les affectent. Le nombre de billons disponibles est malheureusement trop faible pour permettre de vérifier cette hypothèse.

\section{TABLEAU 6}

Corrélations entre la résisfance au choc et les autress caractères Correlation coefficients between impact bending tesfy and the other mechanical properties

Les limites de signification sont de 0,602 au seuil de $5 \%$

0,735 au seuil de $1 \%$

0,847 au seuil de $1 \%$

\begin{tabular}{l|l|l|l|l|l|l|l|l|l|l|}
\hline \hline & PO/VS & RFS & FLR & RCA & DUR & RR & RA & RT & RV \\
\hline CHOC & 0.611 & 0,836 & N.S. & 0.822 & N.S. & 0.729 & N.S. & 0,676 & N.S. \\
\hline
\end{tabular}

2.24. Étude sur les éprouvettes du type III des liaisons existant entre les caractéristiques mécaniques et de retrait et les composantes de la densité.

2.241. Liaisons entre composantes de la densité. - Les tableaux 7,8 et 9 des corrélations intrabillons, interbillons ou totales font ressortir les liaisons existant entre les diverses composantes de la densité :

La densité minimale annuclle est liẻe positivement à l'infradensité, mais pas aux largeurs des accroissements annuels.

La densité maximale augmente en même temps que les largeurs de cernes de façon très significative (matrices de corrélations intrabillons ou totales) puisque les cernes larges ont beaucoup de bois d'été comportant essentiellement des fibres. Par voie de conséquence, I'hétérogénéité s'aggrave lorsque la vitesse de croissance augmente, et les corrélations entre celle-ci et l'infradensité sont élevées dans les trois tableaux de corrélation. 


\section{TABLEAU 7}

Matrice des roefficients de corrélation intrabillons

Correlation coefficients within bolts

Les limites de signification sont de 0,143 au seuil de $5 \%$

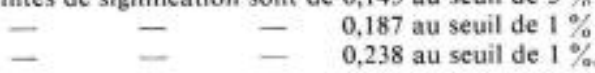

\begin{tabular}{|c|c|c|c|c|c|c|c|c|c|c|c|c|c|c|c|c|}
\hline & RFS & FLR & $\mathrm{RCA}$ & DUR & RR & RA & RT & RV & PO/VS & $\mathrm{dm}$ & DM & $\underset{\mathrm{dm}}{\mathrm{DM}}-$ & LC & $500 \%$ & $\underset{600}{L D^{\prime}}$ at & 700 \\
\hline PO/VS & 0,537 & 0,247 & 0,687 & 0,703 & 0,252 & I & 0,456 & 0,506 & 1 & & & & & & & \\
\hline $\mathrm{dm}$ & 0,344 & 0,204 & 0,336 & 0,378 & 0,219 & 1 & 0,331 & 0,393 & 0,475 & 1 & & & & & & \\
\hline DM & 0.555 & 0,300 & 0,632 & 0.708 & 0,184 & 1 & 0,372 & 0,400 & 0,722 & 0,296 & 1 & & & & & \\
\hline $\mathrm{DM}-\mathrm{dm}$ & 0,247 & 1 & 0,323 & 0,361 & 1 & 1 & 1 & 1 & 0,301 & $-0,486$ & 0,690 & 1 & & & & \\
\hline $\mathrm{LC}$ & 0,378 & I & 0.452 & 0,546 & 1 & t & 0,263 & 0,261 & 0,573 & I & 0.619 & 0,458 & 1 & & & \\
\hline$\%$ LD $>500$ & 0,501 & 0,305 & 0,506 & 0,567 & 0,228 & 1 & 0,369 & 0,425 & 0,620 & 0,815 & 0,553 & 1 & 0,382 & 1 & & \\
\hline$\% \mathrm{LD}>600$ & 0,561 & 0,285 & 0,602 & 0,696 & 0,226 & 1 & 0,377 & 0.425 & 0,702 & 0,533 & 0,809 & 0,336 & 0,592 & 0,798 & 1 & \\
\hline$\%$ LD $>700$ & 0,584 & 0,257 & 0,660 & 0.729 & 0,295 & 1 & 0,427 & 0,507 & 0,752 & 0,381 & 0,864 & 0,501 & 0,649 & 0,596 & 0,809 & 1 \\
\hline
\end{tabular}


TABLEAU 8

Matrice des coefficients de corrélation interbillons

Correlations coefficients between bolts

Les limites de signification sont de 0,878 au seuil de $5 \%$

- _ _ _ 0,959 au seuil de $1 \%$

0,991 au seuil de $1 \%$

\begin{tabular}{|c|c|c|c|c|c|c|c|c|c|c|c|c|c|c|c|c|}
\hline & RFS & FLR & $\mathrm{RCA}$ & DUR & $\mathrm{RR}$ & RA & RT & RV & $\mathrm{PO} / \mathrm{VS}$ & $\mathrm{dm}$ & DM & $\underset{\mathrm{dm}}{\mathrm{DM}-}$ & LC & $500 \%$ & $\underset{600}{L_{0}}$ & 700 \\
\hline $\mathrm{PO} / \mathrm{vS}$ & 1 & 1 & I & I & 1 & 1 & 1 & 1 & 1 & & & & & & & \\
\hline $\mathrm{dm}$ & 1 & 1 & 1 & I & 1 & 1 & 1 & 1 & 1 & 1 & & & & & & \\
\hline DM & 1 & 1 & 1 & 0,927 & 1 & 1 & 1 & 0,904 & 0,926 & 1 & 1 & & & & & \\
\hline $\mathrm{DM}-\mathrm{dm}$ & 0,920 & 1 & 1 & 0,931 & 0,894 & t & 1 & 1 & 0,928 & 1 & 0,889 & 1 & & & & \\
\hline LC & 1 & I & 1 & 1 & l & I & 0,960 & 1 & I & 1 & 1 & I & 1 & & & \\
\hline$\%$ LD $>500$ & 1 & I & 1 & 1 & 1 & 1 & 0,940 & f & 1 & 1 & 1 & I & I & 1 & & \\
\hline$\% \mathrm{LD}>600$ & 1 & l & I & 0,979 & 1 & 1 & 1 & 0,985 & 1 & 1 & 0,958 & 0,898 & 1 & 1 & 1 & \\
\hline$\% \mathrm{LD}>700$ & 0,908 & 1 & 1 & 0,973 & 0,894 & 1 & 1 & 0,917 & 0,932 & I & 0,965 & 0,974 & 1 & I & 0,966 & 1 \\
\hline
\end{tabular}


TABLEAU 9

Matrice des coefficients de corrélation totale

Correlation coefficients for all the samples

Les limites de signification sont de 0,142 au seuil de $5 \%$

- - _ $\quad 0,186$ au seuil de $1 \%$

- $\quad$ - $\quad$ - $\quad 0,236$ au seuil de $1 \%$

\begin{tabular}{|c|c|c|c|c|c|c|c|c|c|c|c|c|c|c|c|c|}
\hline & RFS & FLR & RCA & DUR & R R & RA & RT & RV & PO/VS & $\mathrm{dm}$ & DM & $\underset{\mathrm{dm}}{\mathrm{DM}}$ & LC & $500^{\circ}$ & $\underset{600}{L D}>a$ & 700 \\
\hline PO/VS & 0,658 & 0,241 & 0,626 & 0,734 & 0,483 & $-0,182$ & 0,429 & 0,584 & 1 & & & & & & & \\
\hline $\mathrm{dm}$ & 0,181 & 0,176 & 1 & 0,314 & 0,142 & I & 0,384 & 0,366 & 0,303 & 1 & & & & & & \\
\hline DM & 0,659 & 0.291 & 0,629 & 0,794 & 0,426 & 1 & 0,462 & 0.580 & 0,793 & 0,317 & $t$ & & & & & \\
\hline $\mathrm{DM}-\mathrm{dm}$ & 0,517 & 0,162 & 0,534 & 0.556 & 0,317 & $-0,149$ & 0,185 & 0,313 & 0,564 & -0.380 & 0,755 & 1 & & & & \\
\hline LC & 0,372 & 1 & 0,374 & 0,574 & 0,205 & 1 & 0,432 & 0,430 & 0,516 & 0,206 & 0,634 & 0,476 & 1 & & & \\
\hline$\%$ LD $>500$ & 0,465 & 0.251 & 0,418 & 0,605 & 0,312 & 1 & 0,486 & 0,537 & 0,526 & 0,802 & 0,622 & 1 & 0,502 & 1 & & \\
\hline$\%$ LD $>600$ & 0,714 & 0,241 & 0,707 & 0,815 & 0,504 & l & 0,480 & 0,640 & 0,761 & 0,443 & 0,869 & 0,541 & 0,652 & 0,782 & 1 & \\
\hline$\%$ LD $>700$ & 0,727 & 0,261 & 0.707 & 0,824 & 0,535 & l & 0,474 & 0,655 & 0,811 & 0,317 & 0,903 & 0,662 & 0,635 & 0,613 & 0,874 & 1 \\
\hline
\end{tabular}


Les pourcentages de longueur radiale de densité supérieure à 600 et $700 \mathrm{~g} / \mathrm{dm}^{3}$ varient comme l'infradensité, la densité maximale, et les largeurs de cernes.

Ces résultats traduisent une tendance générale à l'intérieur de chaque billon, ou de billon à billon, mais il est possible, comme dans le cas de l'étude des arbres sur pied par sondages à la tarière, de trouver des exceptions individuelles à cette tendance. C'est le cas pour l'arbre qui a fourni le billon 104 dont la matrice des coefficients de corrélation ne laisse apparaitre aucune liaison entre largeur de cerne et infradensité, au contraire de tous les autres.

2.242. Liaisons entre qualité du bois et composantes de la densité. - Les rapports en question sont traduits par les coefficients de corrélation qui apparaissent aux intersections des 8 colonnes de gauche et des 8 lignes des tableaux de corrélation; sur le tableau des corrélations intrabillons, la résistance à la flexion statique est en liaison positive avec les 8 variables composantes de la densité, la corrélation la plus forte étant obtenue avec le pourcentage de longueur de densité supérieure à $700 \mathrm{~g} / \mathrm{dm}^{3}(r=0,584$ pour 190 degrés de liberté); ce résultat est confirmé sur le tableau des corrélations interbillons $(r=0,908$ pour 3 degrés de liberté) où en outre se manifeste une liaison flexion statique et hétérogénéité ( $r=0,920$ pour 3 degrés de liberté) comparable à ce que l'on avait déjà pu trouver dans une étude antérieure chez le douglas (KELER, 1968).

La flèche à la rupture est liée positivement à la densité maximale et au pourcentage de longueur oủ la densité dépasse $500 \mathrm{~g} / \mathrm{dm}^{3}$, mais seulement à l'intérieur des billons.

Il en est de même pour la résistance en compression axiale qui est bien corrélée à l'infradensité, ainsi qu'au pourcentage des $700 \mathrm{~g} / \mathrm{dm}^{3}$.

La dureté présente de trés forts coefficients de corrélation, à la fois à l'intérieur des billons et entre les billons, avec la densité maximale, les pourcentages à $600 \mathrm{et} 700 \mathrm{~g} / \mathrm{dm}^{3}$ et I'hétérogénéité; elle est d'ailleurs liée aux largeurs de cernes $(r=0,546$ pour 190 degrés de liberté) dans la matrice des corrélations intrabillons.

Des fortes densités sous quelque forme qu'elles se manifestent et une largeur de cerne élevée sont à l'origine de fortes résistances mécaniques dont certaines, comme la dureté, ne sont d'ailleurs pas recherchées dans le cas d'une utilisation en tranchage, ou encore d'une hétérogénéité elle aussi défavorable dans la plupart des cas.

Cette interaction défavorable entre largeur de cernes et densités élevées agit aussi sur le retrait radial, et surtout sur le retrait tangentiel qui est toujours en corrélation positive avec la vitesse de croissance et le pourcentage à $700 \mathrm{~g} / \mathrm{dm}^{3}$. Le retrait axial semble ne dépendre que peu des variables étudiées; il n'est en liaison négative qu'au niveau des corrélations totales avec l'infradensité $(r=-0,182)$ et avec l'hétérogénéité $(r=-0,149)$, alors qu'un même calcul, fait avec cette dernière caractéristique, à partir des carottes de sondage, donne un résultat non significatif. Le retrait volumétrique est fortement lié à la densité maximale et au pourcentage à $600 \mathrm{~g} / \mathrm{dm}^{3}$ (matrices des corrélations intrabillons, interbillons et totales).

Les calculs de corrélations effectués entre les moyennes des éprouvettes du type I ayant subi les essais au choc et les moyennes des éprouvettes du type III provenant des mêmes billons laissent apparaitre (tableau 10) des liaisons significatives, malgré le faible nombre de degrés de liberté disponibles. 
La résistance au choc est liée positivement aux pourcentages à 600 et $700 \mathrm{~g} / \mathrm{dm}^{3}$, à la densité maximale et à l'hétérogénéité ( $r=0,966$, significatif au seuil de $1 \%$ ).

TABLEAU 10

Corrélations entre la résistance au choc (moyennes par billon dex éprouvettes du type I) et les composantes de la densité (moyennes sur les billons correspondants des éprouvettes du type III) Correlation coefficients betwen impact bending tests and density components

Les limites de signification sont de 0.878 au seuil de $5 \%$ - $\quad$ - $\quad$ - $\quad 0.959$ au seuil de $1 \%$

\begin{tabular}{l|c|c|c|c|c|c|c|c}
\hline & PO/VS & $\mathrm{dm}$ & $\mathrm{DM}$ & $\mathrm{DM}-\mathrm{dm}$ & $\mathrm{LC}$ & $\% \mathrm{LD}>500$ & $\% \mathrm{LD}>600$ & $\%$ LD $>700$ \\
\hline Choc & N.S. & N.S. & 0,874 & 0,966 & N.S. & N.S. & 0,907 & 0,957 \\
\hline \hline
\end{tabular}

\subsection{Explication des propriétés mécaniques et du retrait par les composantes de la densité.}

Le tableau 9 des corrélations totales nous a montré les liaisons entre variables à expliquer et variables explicatives. Les valeurs de ses coefficients correspondent aux valeurs des coefficients de corrélation multiple du premier palier d'une régression progressive. Ce sont donc les variables explicatives qui présentent les liaisons les plus élevées avec la variable à expliquer qui apparaitront en premier lieu dans l'équation de régression.

On a conservé I'humidité au retrait (HR) et l'humidité aux essais (HE) comme variables explicatives pour éliminer deux causes de variation indésirables dans les valeurs mesurées. Lorsqu'elles apparaissent dans les équations linéaires, il suffit de considérer que ce ne sont que des facteurs correctifs et que les véritables variables explicatives sont l'infradensité (PO/VS), la densité minimale (dm), la densité maximale (DM), l'hétćrogénéité (DM-dm), la largeur de cernes (LC), les pourcentages de longueur radiale oủ le bois a une densité égale ou supérieure à un seuil donné, soit $\% \mathrm{LD}>500, \% \mathrm{LD}>600$ et $\% \mathrm{LD}>700$.

La prise en compte d'une variable explicative n'est utile que si elle contribue à diminuer de façon significative l'écart-type résiduel; le tableau 11 des valeurs des coefficients de corrélation multiple ne porte que sur ces variables utiles à la régression. Selon les cas, leur nombre varie de 2 à 5, y compris $\mathrm{HR}$ et $\mathrm{HE}$.

2.251. Résistance en flexion statique (RFS) et flèche à la rupture (FLR), - Si l'on met à part $\mathrm{HR}$ et $\mathrm{HE}$, la résistance en flexion statique est surtout liée à $\% \mathrm{LD}>700$ et à $\% \mathrm{LD}>$ 600 , dont la combinaison linéaire permet de rendre compte de $67 \%$ de la variation initiale mesurée sur les 192 éprouvettes.

Au quatrième palier, on peut écrire :

$$
\begin{aligned}
& \mathrm{RFS}_{\mathrm{kg} / \mathrm{em}^{2}}=73+0,155 \% \mathrm{LD}>700+0,224 \% \mathrm{LD}>600 \\
& -3,275 \text { HR } \%-1,291 \mathrm{HE} \%
\end{aligned}
$$




\section{TABLEAU 11}

Regressions progressives sur 192 échantillons

$$
\text { Step by step regression analysis }
$$

Pourcentage de la variation expliquée avant la régression = percentage of explained variation before the regression analysis Pourcentage de la variation expliquèe après la régression - percentage of explained variation after the regression analysis

\begin{tabular}{|c|c|c|c|c|c|c|c|}
\hline \multirow{2}{*}{$\begin{array}{c}\text { Caractère } \\
\text { Rang }\end{array}$} & \multicolumn{5}{|c|}{ Variables introduites } & \multicolumn{2}{|c|}{$\begin{array}{c}\% \text { de la variation } \\
\text { expliquée }\end{array}$} \\
\hline & 1 & 2 & 3 & 4 & 5 & $\begin{array}{l}\text { avant la } \\
\text { régression }\end{array}$ & $\begin{array}{l}\text { après la } \\
\text { régression }\end{array}$ \\
\hline RFS & $\% \underset{0,727}{\operatorname{LD}} 700$ & $\underset{0,794}{\mathrm{HR}}$ & $\% \underset{0,806}{\operatorname{LD}}>600$ & $\begin{array}{c}\mathrm{HE} \\
0,819\end{array}$ & & 53 & 67 \\
\hline FLR & $\underset{0,290}{\mathrm{D}}$ & $\underset{0,347}{\mathrm{LC}}$ & & & & 8 & 12 \\
\hline RCA & $\% \underset{0,707}{\mathrm{LD}}>600$ & $\underset{0,770}{\mathrm{HE}}$ & $\underset{0,805}{\mathrm{HR}}$ & $\underset{0,815}{\mathrm{dm}}$ & $\% \underset{0,822}{\text { L.D }} 700$ & so & 68 \\
\hline DUR & $\% \underset{0,825}{\text { LD }}>700$ & $\% \underset{0,848}{\text { LD }}>600$ & $\begin{array}{c}\text { PO/VS } \\
0,851\end{array}$ & & & 68 & 72 \\
\hline RR & $\% \underset{0,535}{\operatorname{LD}}>700$ & $\underset{0,641}{\mathrm{HR}}$ & $\underset{0,653}{L C}$ & & & 29 & 43 \\
\hline RA & $\begin{array}{c}\text { PO/VS } \\
0,182\end{array}$ & $\% \underset{0,250}{\operatorname{LD}>} 500$ & & & & 3 & 6 \\
\hline RT & $\% \underset{0,486}{\operatorname{LD}} \underset{>}{ } 500$ & $\% \underset{0,534}{L D_{3}}>700$ & $\underset{0,586}{\mathrm{HE}}$ & $\begin{array}{c}\mathrm{HR} \\
0,617\end{array}$ & $\begin{array}{c}\mathrm{PO} / \mathrm{VS} \\
0,631\end{array}$ & 24 & 40 \\
\hline RV & $\% \underset{0,655}{L D} 700$ & $\begin{array}{c}\text { HR } \\
0,733\end{array}$ & $\begin{array}{c}\mathrm{HE} \\
0,759\end{array}$ & $\begin{array}{l}\mathrm{PO} / \mathrm{VS} \\
0,771\end{array}$ & $\underset{0,778}{\mathrm{dm}}$ & 43 & 61 \\
\hline
\end{tabular}


Chez le chêne, la résistance en flexion statique ne dépend donc pas en premier lieu de l'infradensité, comme on avait pu le voir chez le frêne, mais de l'importance des zones à forte densité.

Les corrélations entre FLR et les autres caractéristiques n'ont jamais été élevées dans cette étude. La régression progressive ne conduit qu'à l'introduction significative de deux variables, dans l'ordre D, puis LC, qui ne permettent d'expliquer que $12 \%$ de la variabilité totale. D'autres variables explicatives que celles qui étaient à notre disposition seraient à rechercher pour une meilleure connaissance du phénomène.

Chez le frêne, on avait réussi à rendre compte de plus de $30 \%$ de la variabilité à l'aide d'une combinaison linéaire de D, LC et d'un pourcentage de densité $>800 \mathrm{~g} / \mathrm{dm}^{3}$.

2.252. Résistance en compression axiale (RCA). - C'est $\%$ LD $>600$ qui apparait en première place, puis pour mémoire $\mathrm{HE}$ et $\mathrm{HR}$, et enfin dm et $\% \mathrm{LD}>700$. Les pourcentages ont tous deux un coefficient positif dans la régression, alors que la densité minimale a un effet dépressif sur cette résistance puisqu'elle est précédée d'un coeflicient négatif.

$$
\begin{gathered}
\mathrm{RCA}_{\mathrm{kg} / \mathrm{cm}^{2}}=919+3,55 \% \mathrm{LD}>600+1,05 \% \mathrm{LD}>700 \\
-0,287 \mathrm{dm}_{\mathrm{g} / \mathrm{dm}^{2}}-19,25 \mathrm{HE} \%-2,69 \mathrm{HR} \%
\end{gathered}
$$

Dans le cas du frêne, la compression axiale était liée positivement à l'infradensité et aux très hauts niveaux de densité, et négativement au niveau intermédiaire $\% \mathrm{LD}>800 \mathrm{~g} / \mathrm{dm}^{3}$.

2.253. Dureté (DUR). - Comme nous l'avons indiqué ci-dessus dans la méthodologie, cette caractéristique a été mesurée sous la forme d'une largeur d'empreinte. Plus cette largeur est petite, plus la dureté est grande, et dans l'équation qui traduit la relation entre la largeur d'empreinte et les variables explicatives $\% \mathrm{LD}>700, \% \mathrm{LD}>600$ et $\mathrm{PO} / \mathrm{VS}$, ces dernières sont précédées de coefficients négatifs :

$$
\begin{aligned}
\text { largeur d'empreinte } & =8,85-0,014 \% \mathrm{LD}>700-0,019 \% \mathrm{LD}>600 \\
& -0,002 \mathrm{PO} / \mathrm{VS}_{\mathrm{g} / \mathrm{dm}^{2}}
\end{aligned}
$$

Les trois variables explicatives étant liées, on l'a vu, à la vitesse de croissance, on retrouve indirectement l'influence de cette dernière sur la qualité du bois de chêne. Elles expliquent ici $72 \%$ de la variation totale observée.

Chez le frêne, on avait trouvé comme variables explicatives, en premier lieu $\mathrm{PO} / \mathrm{VS}$, puis $\%$ LD $>800 \mathrm{~g} / \mathrm{dm}^{3}$.

\subsection{Retraits de l'état saturé à l'état sec à l'air (à $10 \%$ d'humidité) $)$}

2.254.1. Retrait axial (RA). - PO/VS et \% LD > 500 expliquent une part très petite de la variation. Rappelons que ce retrait est lié négativement à la flèche à la rupture dans le tableau des coefficients de corrélation interbillons et que nos variables explicatives s'étaient également montrées inefficaces dans l'étude de cette propriété (paragraphe 2251). 


\subsubsection{Retraits radial (RR), tangentiel (RT) et volumétrique (RV).}

C'est chaque fois un pourcentage qui vient en tête des variables explicatives complété, selon le cas, par un autre pourcentage, LC ou dm :

$$
\begin{aligned}
\mathrm{RR} \%= & 7,72+0,023 \% \mathrm{LD}>700-0,289 \mathrm{LC}_{\mathrm{mm}}-0,364 \mathrm{HR} \% \\
\text { RT } \%= & 3,76+0,019 \% \mathrm{LD}>500+0,007 \% \mathrm{LD}>700+0,004 \mathrm{PO} / \mathrm{VS}_{\mathrm{g} / \mathrm{dm}^{3}} \\
& +0,303 \mathrm{HE} \%-0,255 \mathrm{HR} \% \\
\mathrm{RV} \%= & 11,46+0,025 \% \mathrm{LD}>700+0,006 \mathrm{PO} / \mathrm{VS}_{\mathrm{g} / \mathrm{dm}^{3}}+0,004 \mathrm{dm}_{\mathrm{g} / \mathrm{dm} \mathrm{m}^{3}} \\
& +0,262 \mathrm{HE} \%-0,677 \mathrm{HR} \%
\end{aligned}
$$

L'étude de deux éléments anatomiques au moins serait susceptible de donner des indications complémentaires sur le retrait du chêne. Puisque dans chacune des trois régressions on trouve $\% \mathrm{LD}>700$, on peut penser que les retraits radial, tangentiel et volumétrique sont des phénomènes conditionnés par les cellules de forte densité, c'est-à-dire les fibres du bois final et les cellules de parenchyme lignifié des rayons ligneux.

Dans une étude non publiće sur le hêtre, on a trouvé une corrélation significative entre le retrait tangentiel et le volume relatif des gros rayons ligneux, ou encore le nombre de rayons ligneux à l'unité de surface. Le retrait radial et le retrait tangentiel y sont d'autant plus faibles que la surface unitaire d'un rayon ligneux est plus grande.

Quant au retrait axial, il faudrait essayer de l'étudier, non pas seulement à partir des composantes de la densité, mais aussi à l'aide de données caractérisant les membranes cellulaires qu'elles ne prennent qu'imparfaitement en compte.

\section{CONCLUSION}

Les essais destructifs dont il est rendu compte ci-dessus montrent qu'il existe de fortes liaisons entre les divers critères de densité (infradensité et composantes de la densité) et les autres caractéristiques physiques (retraits) ou mécaniques.

Dans certains cas, le pourcentage d'explication obtenu en régression progressive est tel qu'il est possible de prévoir dans une large mesure la façon dont le bois résistera aux contraintes qui pourront lui être appliquées, à partir de combinaisons linéaires de paramètres densitométriques.

On peut penser que l'on disposera toujours en France d'approvisionnements suffisants en bois de chêne dur et nerveux à caractéristiques mécaniques élevées; en revanche, le déficit en bois tendre apte aux utilisations les plus nobles, comme le tranchage ou l'ébénisterie fine, ne cesse de s'aggraver; il est donc raisonnable, dans des forêts comme celle de Tronçais où cela est possible, de rechercher avant tout la production de bois à faible densité, à faible rétractibilité, à faible hétérogénéité et à faible dureté, les quatre allant généralement de pair, même s'il doit en résulter une diminution des autres caractéristiques mécaniques.

Cet objectif étant défini, le présent travail a permis de mettre en évidence un certain nombre de points importants :

- lorsque l'on compare entre elles des parcelles différentes d'âges variés, celles qui 
bénéficient de la croissance la plus rapide (ce sont également les plus jeunes) produisent le bois le plus médiocre.

- lorsque l'on compare entre eux des individus d'âge très voisin au sein d'une même parcelle, on constate, contrairement à ce qui était généralement admis jusqu'ici, que les individus ayant les accroissements les plus larges ne sont pas nécessairement ceux dont la densité est la plus forte; ceci tient à ce que la largeur de la zone initiale elle-même est variable d'un individu à un autre, et aussi à ce que, dans le bois final, la richesse relative, en fibres d'une part, en petits vaisseaux et en parenchyme périvasculaire d'autre part, est loin d'être uniforme; il existe en fait dans toutes les parcelles un très large éventail de plans ligneux qu'il paraît extrêmement difficile d'expliquer par des microvariations des facteurs édaphiques ou de la concurrence.

Cette variabilité est telle qu'on est amené à lui attribuer une origine génétique; on ne pourra le vérifier que lorsque l'on disposera de plantations comparatives de descendances, mais il n'est pas illogique de penser à une influence possible de l'hérédité, étant donné que la forêt de Tronçais est en partie issue de régénérations artificielles ayant eu pour effet de briser les cercles de consanguinité, que la longueur des révolutions y a réduit au minimum les mélanges ultérieurs de gènes, et que les possibilités de dissémination naturelle de graines lourdes, comme les glands, se limitent à celles qui résultent de l'action de la faune forestière.

- un dernier enseignement trẻs utile peut être tiré de cette expérience : il concerne I'intérêt de la régularité des accroissements annuels : chaque fois que se produit une accélération de la croissance du chêne, la qualité se dégrade; il y a done un risque certain à le faire pousser plus vite par des moyens culturaux, et il semblerait plus raisonnable de chercher à améliorer à terme la productivité des chênaies par la sélection d'individus à croissance rapide, mais à caractéristiques technologiques satisfaisantes.

Si l'on se souvient de l'importance que présente au contraire le traitement en matière de qualité du bois de hêtre (Pol.ge, 1973), on peut dire que les deux principales espèces feuillues françaises se comportent de façon totalement différente; l'intérêt de la sylviculture traditionnelle des chênaies se trouve confirmé par le présent travail, alors que des techniques beaucoup plus révolutionnaires de gestion semblent pouvoir être appliquées aux hêtraies, sans répercussion fâcheuse sur la qualité du bois.

Recu pour publication en mai 1973.

\section{REMERCIEMENTS}

Ce travail n'a été rendu possible que grâce à l'aide de M. Ror, Chef du Centre de Gestion de l'Office National des Forêts de Moulins, qui a mis à notre disposition les échantillons nécessaires aux essais de caractére destructif et qui, de plus, nous a fourni tous les renseignements utiles concernant les diverses stations étudiées.

Nous lui en sommes très reconnaissants, et nous tenons également à remercier vivement la Station de Biométrie du C.N.R.F., et notamment M. MiLLIER qui a pris en charge le traitement statistique des très nombreuses données recueillies. 


\section{SUMMARY}

\section{WOOD QUALITY AND RINGWIDTH IN TRONÇAIS FOREST}

The following informations has been gathered from an experiment in the " Tronçais " forest where 125 increment cores and 740 standardized samples were studied.

When we compare different plots the wood density increases when the ringwith increases (due principally to an age effect) - fig. 4 .

Within a plot this positive correlation is not so clear and never significant, i.e. it is always possible to find trees with large rings and rather low wood specific gravity (fig. 1 and 2 ).

When the growth of a given tree becomes faster the wood density increases (fig. 5 and 6 ).

The measurements made on non destructive as well as on standardized samples show that wood density is positively correlated with mechanical strength and with shrinkage (table 5, 7, 8 and 9).

For veneer production wood with low hardness and low shrinkage is required, that means that for this profitable utilization high density is to be considered as a defect.

It follows that in oak forest like " Troncais » where veneer production is the main objective, it is dangerous to accelerate growth by silvicultural means and a higher volume production is only to be expected from the selection of individuals with large rings but also, which is not incompatible, with low wood density.

\section{ZUSAMMENFASSUNG}

\section{HOLZQUALITAT UND JAHRRINGBREITE IM FORET DOMANIALE DE TRONÇAIS}

Vergleicht man mehrere Parzellen, so kann man allgemein festellen, dass mit zunehmender Wuchsleistung, die Rohdichte, die Härte und die Schwindung des Holzes der Traubeneichen gleichfalls zunehmen. Vergleicht man jedoch verschiedene Bäume innerhalb einer Parzelle, so kann man nicht immer eine Antinomie zwischen Raschwüchsigkeit und guter Holzqualität feststellen.

Desweiteren zeigt sich, dass, sobald eine Eiche breite Jahrringe ausbildet, auch die Rohdichte automatisch zunimmt und somit die Fournirholzqualität beeinträchtigt.

\section{RÉFÉRENCES BIBLIOGRAPHIQUES}

DE Saint Vaulky, 1969, - L'individualisation précoce des arbres d'avenir. R.F.F., mars-avril, p. 83.

DuCEll.ter, 1930. - La forèt de Bellème et une nouvelle méthode d'éclaircie. Revae des Eaux et Forërs, avril, p. 263.

Ducellere, 1931. - L'éclaircie méthodique de Bellème. R.E.F. juillet, p. 567.

KELLer R., 1968. - Des caractéristiques nouvelles pour l'étude des propriétés mécaniques des bois : les composantes de la densité, Ann. Sci. foress., 25 (4), 237-249.

Lacaze J.-F. et Polge. H., 1970. - Relations phénotypiques au stade juvénile entre la densité du bois et divers caractères phènologiques et de vigueur chez Picea abies Karst. Ann. Sci. forest, 27 (3), 231242.

LORNE R., 1956. - A la recherche de la qualité et du gros diamètre dans les futaies de chếne. R.F.F., novembre, p. 754.

LORNE R.. 1959, - Étude quantitative sur les éclaircies dans les peuplements de chène de qualité, R.F.F., novembre, p. 746.

LORNE R., 1961, - La sylviculture de I'avenir, R.F.F, juillet, p. 503.

Marotre P., 1956. - L'industrie du tranchage en France. R.F.F., juillet, p. 495. 
Polge H., 1963. - Contribution à l'étude de la qualité du bois des principales essences résineuses exotiques utilisées dans les reboisements français. Annales de l'Ecole Nationale des Eaux et Foréts et de la Station de Recherches et Expériences, tome XX, fascicule 3, 403-467.

Polge H., 1968. - Tests précoces de la qualité du bois sur 25 provenances d'Abies grandis. Ann. Scl. forest. 25 (1) 3-23.

Polge H. et Keller R., 1973. - Influence de l'élagage de branches vivantes sur la structure des accroissements annuels et sur quelques caracteristiques du bois de douglas et de grandis. Ann. Sci. forest. 30 (2) $127-140$.

Polge H., 1973. - Etat actuel des recherches sur la qualité du bois de hêtre. Bulletin Technique de IO N.N.F., $n^{*} 4$.

Thizrcelin F., 1970. - Tardiveté du débourrement et densité du bois dans une population adulte de Picea abies Karst. Ann. Sci. forest. 27 (3), 243-254.

VANSflow, 1956. - Die Geschichte der Forsteinrichtung in den Bayerischen Staatswaldungen im Spessart. Mitteilungen aus der Staatsforstverwaltung Bayerns 28 Heft, p. 6-178.

VENET J., 1967 a. - Sylviculture des foréts de chène de tranchage. R.F.F., décembre, p. 746.

VENET J., 1967 b. - Le chẻne de tranchage et les utilisateurs. R.F.F., octobre, p. 586.

VENET J., 1968, - Pratique de la pré-désignation des arbres de place, R.F.F., mars, p. 158.

VENET J., 1973. - Identification et classement des bois français. E.N.G.R.E.F. Nancy, à paraitre.

VINEY R. et PARDE J., 1960. - Pour le dossier des chénes de qualité. R.F.F., janvier p. 44.

Schulz H., 1959. - Untersuchungen über Bewertung und Gütemerkmale des Eichenholzes aus Verschiedenen Wuchsgebieten. Sauerländer's Verlag Editeur, Francfort/Main. 